\title{
Title: Heterogeneous Responses of Nucleus Incertus Neurons to Corticotropin- Releasing Factor and Coherent Activity with Hippocampal Theta Rhythm in the Rat
}

Authors: Sherie Ma, ${ }^{1,2}$ Anna Blasiak, ${ }^{3}$ Francisco E. Olucha-Bordonau, ${ }^{4}$ Anthony J.M. Verberne $^{2}$ and Andrew L. Gundlach ${ }^{1,5}$

Affiliations: 1. The Florey Institute of Neuroscience and Mental Health, The University of Melbourne, Victoria 3010, Australia; 2. Department of Medicine, Austin Health, The University of Melbourne, Heidelberg, Victoria 3084, Australia; 3. Department of Neurophysiology and Chronobiology, Jagiellonian University, PL- 30-387 Krakow, Poland; 4. Fundación para la investigación Hospital Clínico de Valencia INCLIVA, CIBERSAM and Departamento de Anatomía y Embriología Humana, Facultad de Medicina, Universidad de Valencia, 46010 Valencia, Spain; 5. Department of Anatomy and Neuroscience, The University of Melbourne, Victoria 3010, Australia.

Keywords: nucleus incertus, corticotropin-releasing factor, electrophysiology

Running title: Electrophysiology of nucleus incertus neurons

Total number of words: 10,253

Corresponding author: Dr Sherie Ma, The Florey Institute of Neuroscience and Mental Health, The University of Melbourne, Victoria 3010, Australia. E-mail:

sherie.ma@florey.edu.au

Table of contents category: Neuroscience

This is an Accepted Article that has been peer-reviewed and approved for publication in the The Journal of Physiology, but has yet to undergo copyediting and proof correction. Please cite this article as an "Accepted Article"; doi:10.1113/jphysiol.2013.254300. 


\section{Key points summary}

1. The nucleus incertus (NI) is a stress and arousal responsive, hindbrain region involved in ascending control of septohippocampal theta rhythm.

2. NI neurons express high levels of the neuropeptide relaxin-3 and corticotropin-releasing factor (CRF) receptor-1 (CRF-R1).

3. We report the first in-depth characterisation of NI neurons, using in vivo and in vitro electrophysiological techniques, which reveal a population of relaxin-3-containing NI neurons that are activated by CRF via postsynaptic CRF-R1 and a non-relaxin-3 neuron population that is inhibited or unaffected by CRF.

4. Relaxin-3 NI neurons exhibit strong phase-locked firing with the ascending phase of hippocampal theta oscillations.

5. These findings suggest the NI is a heterogeneous neuronal population and key site of CRF action with the capacity to modulate cognition in response to stress.

Summary word count: 126 


\begin{abstract}
The nucleus incertus (NI) of the rat hindbrain is a putative node in the ascending control of the septohippocampal system and hippocampal theta rhythm and is stress and arousal responsive. NI contains GABA neurons that express multiple neuropeptides including relaxin-3 (RLN3) and neuropeptide receptors including corticotropin-releasing factor receptor-1 (CRF-R1), but the precise anatomical and physiological characteristics of NI neurons are unclear. Therefore, we examined the firing properties of NI neurons and their responses to CRF, the correlation of these responses with occurrence of relaxin-3, and NI neuron morphology in the rat. Most NI neurons excited by icv CRF infusion were RLN3positive (9/10), whereas all inhibited cells were RLN3-negative (8/8). The spontaneous firing of RLN3 ( $n=6$ ) but not non-RLN3 neurons $(n=6)$ was strongly modulated and phase-locked with the initial ascending phase of hippocampal theta oscillations. In brain slices, $79 \%$ of recorded NI neurons (15/19) displayed excitatory responses to CRF, which uniformly increased action potential frequency and membrane potential depolarisation in the presence of tetrodotoxin, indicating a direct, postsynaptic action of CRF on NI neurons. This excitation was associated with reduction in the slow component of afterhyperpolarisation and a strong depolarisation. Quantitative analysis in naïve rats of validated CRF-R1, RLN3 and neuronal nuclear antigen $(\mathrm{NeuN})$ immunoreactivity revealed $52 \%$ of NI neurons as CRF-R1 positive, of which $53 \%$ were RLN3 positive; while $48 \%$ of NI neurons lacked CRF-R1 and RLN3. All RLN3 neurons expressed CRF-R1. CRF neurons that projected to the NI were identified in lateral preoptic hypothalamus, but not in paraventricular hypothalamus, bed nucleus of stria terminalis or central amygdala. Our findings suggest NI is an important site for CRF modulation of hippocampal theta rhythm via effects on GABA/RLN3 transmission.
\end{abstract}

\footnotetext{
Abbreviations list

AHP, afterhyperpolarisation; BNST, bed nucleus of the stria terminalis; CRF, corticotropinreleasing factor; CRF-R1, corticotropin-releasing factor receptor-1; FG, fluorogold (retrograde tracer); icv, intracerebroventricular; -IR, immunoreactivity; LC, locus coeruleus; LPO, lateral preoptic area; NeuN, neuronal nuclear antigen; NI, nucleus incertus; RLN3, relaxin-3.
} 


\section{Introduction}

Arousal requires an 'ascending reticular activating system' comprised of brainstem, hypothalamic and forebrain neural networks and lesion/disruption impairs arousal, promotes sleep or induces coma (Moruzzi \& Magoun 1949; Lu et al. 2006; Espana \& Scammell 2011; Fuller et al. 2011). Corticotropin-releasing factor (CRF), a primary stress peptide, is rapidly up-regulated and released from neural populations to mediate adaptive responses to stress (Koob \& Bloom 1985; Aguilera \& Liu 2012). The neural circuits underlying these effects are still being identified and defined.

The nucleus incertus (NI) is a cluster of large neurons adjacent to the $4^{\text {th }}$ ventricle in the midline tegmentum. It was first characterised anatomically and neurochemically in the rat as a site of dense CRF binding and activation (Potter et al. 1994; Bittencourt \& Sawchenko 2000). NI efferent and afferent connections suggest it integrates information relating to behavioural planning with inputs from prefrontal cortex, lateral habenula, and septohippocampal and oculomotor systems (Goto et al. 2001; Olucha-Bordonau et al. 2003). NI projection targets suggest a role in modulating prefrontal cortex and septohippocampal activity, associated with arousal and learning processes (Goto et al. 2001; Olucha-Bordonau et al. 2003). Further anatomical and physiological evidence supports an influence of NI on the septohippocampal pathway, including a vital role in mediating brainstem-induced hippocampal theta rhythm $(4-12 \mathrm{~Hz})$ that underlie mnemonic processing (Nunez et al. 2006; Teruel-Marti et al. 2008; Cervera-Ferri et al. 2011; Ryan et al. 2011).

NI neurons contain GABA and various peptides, including cholecystokinin, neurotensin and neuromedin-B (Kubota et al. 1983; Sutin \& Jacobowitz 1988; OluchaBordonau et al. 2003; Ryan et al. 2011). The NI is also the primary source of the highly conserved relaxin-family peptide, relaxin-3 (RLN3) in the rat (Burazin et al. 2002; Tanaka et al. 2005; Ma et al. 2007), mouse (Bathgate et al. 2002; Smith et al. 2010) and macaque (Ma et al. 2009b). Punctate RLN3-immunoreactivity (-IR) is observed in the cytoplasm of GABAergic NI neurons and in nerve axons, fibres and terminals throughout the brain, consistent with anterograde-tracer labelled NI projections and the distribution of its cognate receptor, RXFP3 (Ma et al. 2007). We have demonstrated that RLN3/RXFP3 signalling in the medial septum contributes to hippocampal theta rhythm and spatial memory in rats (Ma et al. 2009a). Furthermore, NI RLN3 neurons co-express CRF-R1 and are activated, as reflected by increased Fos-IR, following intracerebroventricular (icv) CRF infusion (Tanaka et al. 2005); and repeat forced-swim stress in rats elevated RLN3 mRNA and heteronuclear RNA 
and peptide, an effect attenuated by systemic pre-treatment with the CRF-R1 antagonist, antalarmin (Banerjee et al. 2010). Thus, this newly identified neuropeptide system is thought to mediate stress response and arousal and the NI is a potentially important brainstem site for CRF action to modulate arousal and cognitive processes in responses to neurogenic stress (Ryan et al., 2011).

In this study, we have characterised the interaction of CRF with NI/RLN3 neurons by quantifying their relative levels and co-expression of CRF-R1- and RLN3-IR, identifying their endogenous CRF innervation, examining their responses to CRF in vivo and in vitro and their coherence firing with hippocampal theta oscillations. 


\section{Materials and Methods}

\section{Ethical approval}

Animal experiments, including all surgical procedures, were conducted with the approval of the Florey Institute of Neuroscience and Mental Health and Austin Health Animal Welfare Committees and according to ethical guidelines issued by the National Health and Medical Research Council of Australia (Melbourne), and with the approval of the Ethics Committee of the University of Valencia (Valencia) and animal care guidelines of the European Community Council Directive of 24 November 1986 (86/609/EEC) (Valencia and Krakow). The authors have read, and these experiments comply with, the policies and regulations of The Journal of Physiology (Drummond 2009).

In vivo unit recordings of NI neurons and response to CRF

Adult male Sprague-Dawley rats (300-400 g; $\mathrm{n}=75$; Australian Research Centre, Canning Vale, WA, Australia) were anaesthetised with isoflurane (Delvet, Seven Hills, NSW, Australia) by inhalation in a closed chamber, tracheotomised and artificially ventilated with $100 \%$ oxygen containing $1.7-2 \%$ isoflurane (50-60 breaths $/ \mathrm{min} ; 1 \mathrm{ml} / 100 \mathrm{~g}$ ). Adequacy of anaesthetic depth was verified regularly by absence of an eye blink response following corneal probing and of a withdrawal response to firm paw pinch. A servo-controlled heating pad (Yellow Springs Instruments, Yellow Springs, OH, USA) was used to maintain core temperature between $36-38^{\circ} \mathrm{C}$. Catheters were implanted into the right carotid artery and right jugular vein for measurement of arterial pressure and heart rate and for intravenous administration of urethane, respectively.

The head was then fitted into a stereotaxic frame (Kopf Instruments, Tujunga, CA, USA) and tilted downwards (incisor bar $-12.5 \mathrm{~mm}$ ) to facilitate vertical penetration. The scalp was incised and overlying fascia retracted from the skull. A large trephine hole over the cerebellum was made to allow penetration of a recording electrode to the locus coeruleus (LC) and midline NI. Urethane anaesthesia (1.4 g/kg, i.v.; Sigma-Aldrich, Sydney, Australia) was initiated $1 \mathrm{~h}$ prior to unit recordings and supplemental urethane was administered to maintain depth of anaesthesia. Single-unit neural activity was recorded using glass micropipettes (2 mm OD; Harvard Apparatus, Kent, England), which were pulled to a fine tip using an electrode puller (Narishige, Tokyo, Japan) and filled with $2 \%$ biotinamide (Invitrogen-Molecular Probes, Sydney, Australia) in 0.5 M sodium acetate; electrode impedance was 10-25 M $\Omega$. Due to the small, compact size of the NI, the most efficient way to target the nucleus was to firstly locate LC and the mesencephalic nucleus of the trigeminal 
nerve (Me5) and then moving medially. Recordings were made using an intracellular amplifier in bridge mode (Intra 767; World Precision Instruments, Sarasota, FL, USA) and filtered (400 Hz to $4 \mathrm{kHz}$ bandpass) using a window discriminator (Fintronics, Orange, CT, USA). A hydraulic micropositioner (Kopf Instruments) was used to advance the electrode to the region of the LC and Me5 (AP, -3.1 mm; ML, $1.4 \mathrm{~mm}$; DV, -5.5 to $6.3 \mathrm{~mm}$ from lambda), where neurons displayed increased firing to contralateral paw pinch (Elam et al. 1986) and lower jaw depression (Minkels et al. 1991), respectively. Following verification of LC and Me5, the electrode was moved to the midline to record NI neurons (DV, -6 to $6.5 \mathrm{~mm}$ ). Another small trephine hole was drilled in the skull to allow placement of a concentric bipolar stimulation electrode (Kopf Instruments) into the medial septum ( $5^{\circ}$ angle; AP, -1.4 $\mathrm{mm}$; ML, $1.0 \mathrm{~mm}$; DV, -6.5 to $7 \mathrm{~mm}$ from bregma). Location of the electrode in the medial septum was verified by an increase in arterial pressure $(\sim 15 \mathrm{~mm} \mathrm{Hg})$ in response to electrical stimulation $(200 \mu \mathrm{A} ; 50 \mathrm{~Hz} ; 0.5 \mathrm{~ms})$, as opposed to a decrease in arterial pressure in response to stimulation of the lateral septum (Calaresu \& Mogenson 1972). The recording microelectrode was advanced into the NI while electrical stimulation $(0.5 \mathrm{~Hz} ; 0.5 \mathrm{~ms}$ pulse width; 300-500 $\mu \mathrm{A}$ ) was applied to the medial septum. Action potentials generated by NI neurons were amplified $(\times 1000)$ and filtered $(400-4000 \mathrm{~Hz})$ using an extracellular single unit amplifier and window discriminator (Fintronics) and monitored using an audio amplifier and oscilloscope (Tektronix Inc., Beaverton, OR, USA). Data was acquired using a Power 1401 data acquisition interface and Spike 2 data acquisition software (Cambridge Electronic Design, Cambridge, UK). The antidromic nature of constant latency spikes elicited by stimulation of the medial septum was verified using the collision test (Lipski 1981). Orthodromic spikes were verified using peri-stimulus time histograms of $>100$ consecutive stimulation sweeps.

Following recording, single neurons were juxtacellularly labelled with biotinamide (Pinault 1996). Briefly, positive rectangular pulses (2-8 nA; $200 \mathrm{~ms}$ pulse width; $0.5 \mathrm{~Hz}$ ) were applied until the single-unit activity became robustly entrained by the pulses for $0.5-10$ min, where single-unit entrainment resulted in just one neuron being labelled with biotinamide. Spike shape and amplitude were monitored throughout the procedure to ensure that the neuron entrained/labelled was the same neuron recorded. Only one attempt at labelling was made on each side of the NI to avoid ambiguity in subsequent identification of the neuron. In separate experiments where local field potentials (LFP) of the hippocampus were recorded concurrent with unit recordings in the NI, a wide-field monopolar electrode 
was implanted on top of the hippocampus (AP, $4 \mathrm{~mm}$; ML, $-3 \mathrm{~mm}$; DV, cortical surface) ipsilateral to the recording side of the NI and on the frontal crest as a reference. Hippocampal LFP signal was filtered between $0.3-300 \mathrm{~Hz}$, sampled at $1 \mathrm{kHz}$, and data acquired using a Power 1401 and Spike2 system (Cambridge Electronic Design).

\section{Statistical analyses of in vivo recording data}

Unit activity was analysed for average firing rate (spikes/s) and duration of spikes (measured from the peak of initial positive phase of the action potential to the peak of its subsequent negative phase). Rhythmic firing was indicated by sinusoidal-like density peaks in the autocorrelation histogram. The power spectrum of the EEG was computed using fast Fourier transformation over $0-25 \mathrm{~Hz}$ with $0.1 \mathrm{~Hz}$ resolution to detect hippocampal theta oscillation epochs of greater than $10 \mathrm{~s}$. Each spike was assigned an angle relative to trough $\left(0^{\circ}, 360^{\circ}\right)$ or peak $\left(180^{\circ}\right)$ of theta oscillations using Spike 2 software by fitting a sinewave to surrounding oscillations and calculating angle of the spike ( $18^{\circ}$ bins) from the sinewave angular velocity. Modulation in phase with theta oscillations was tested for significance using Rayleigh's uniformity test (Zar 2010), as described (Fuentealba et al. 2008; Bienvenu et al. 2012). If the null hypothesis that there is no mean population direction/modulation was rejected $(P<0.05)$, phase modulation homogeneity within groups was tested with Moore's non parametric test $(P<0.05)$ and Batschelet's method of calculating population mean angle (Zar 2010). The length $r$ of the normalised vector determined modulation depth.

\section{Histological analyses of juxtacellularly-labelled cells}

At the end of each experiment, rats were deeply anesthetised with $4 \%$ isoflurane and transcardially perfused with $300 \mathrm{ml}$ of ice-cold phosphate-buffered saline (PBS) and $500 \mathrm{ml}$ 4\% formaldehyde in PBS (made from paraformadehyde), as described (Ma et al. 2007). Rats were then decapitated, and brains were dissected and submerged in 30\% sucrose in PBS for $48 \mathrm{~h}$ at $4^{\circ} \mathrm{C}$. Coronal sections $(40 \mu \mathrm{m})$ through the entire NI were cut on a cryostat at $-16^{\circ} \mathrm{C}$ (Cryocut 1800; Leica Microsystems, Heerbrugg, Switzerland) and collected free-floating into PBS just prior to histochemical processing.

To neurochemically phenotype juxtacellularly-labelled cells, sections were incubated in 1:500 dilution of streptavidin-conjugated Dylight488 IgG (Jackson ImmunoResearch Laboratories Inc, West Grove, PA, USA) for $2 \mathrm{~h}$ at room temperature followed by $3 \times 5 \mathrm{~min}$ washes in PBS. Sections were mounted onto glass slides and coverslipped with DAKO fluorescent mounting medium (DAKO Australia, Campbellfield, VIC, Australia) and labelled 
cells were visualised using a Leica DM LB2 microscope (Leica Microsystems, Wetzlar, Germany). Sections containing labelled cells and dendrites were removed from the slide and further processed for RLN3 immunostaining. Sections were washed $3 \times 10 \mathrm{~min}$ and incubated in 1:1000 dilution of monoclonal mouse anti-RLN3 (HK4-144-10; Kizawa et al. 2003; Tanaka et al. 2005) with 2\% NGS in PBS containing 0.1\% Triton X-100 overnight at $4^{\circ} \mathrm{C}$. The monoclonal antibody against RLN3 was raised against an N-terminal region of the mature RLN3 peptide conserved in rat and human. The specificity of this antibody has been described, where immunostaining was completely abolished by preabsorption with native peptide in rat brain (Kizawa et al. 2003; Tanaka et al. 2005) and RLN3 gene knock-out mice (Watanabe et al. 2011). Sections were then washed $3 \times 10 \mathrm{~min}$ and incubated in 1:200 dilution of donkey anti-mouse conjugated DyLight549 IgG (Jackson ImmunoResearch Laboratories) for $2 \mathrm{~h}$ followed by $3 \times 10$ min washes. Sections were re-examined on an Olympus FV1000 laser scanning confocal microscope system (Olympus Imaging, Melbourne, VIC, Australia). Samples were scanned using $63 \times$ magnification and photographed sequentially to collect light emitted as red or green fluorescence. Images were adjusted for brightness and contrast and merged using ImageJ (National Institutes of Health, Bethesda, MD, USA). Only labelled cells that were clearly distinguishable from the background were included in the study.

To visualise the somatodendritic and axonal architecture of labelled neurons, sections containing biotinamide-filled cells were further processed with a permanent reaction product, nickel-enhanced 3,3'-diaminobenzidine tetrahydrochloride (Ni-DAB), as described (Mallet et al. 2012). Brain sections were incubated overnight at room temperature in 1:100 avidin-biotin complex solution (Vectastain Elite, Vector Laboratories, Burlingame, CA, USA) in 0.1 M PBS. Following $3 \times 10$ min washes PBS, sections were incubated in $0.05 \mathrm{M}$ Tris buffer $(\mathrm{pH}$ 8.0) containing hydrogen peroxide $(0.002 \% \mathrm{w} / \mathrm{v}), 3,3$ '-diaminobenzidine tetrahydrochloride $(0.025 \% \mathrm{w} / \mathrm{v}$; Sigma-Aldrich) and nickel ammonium sulphate $(0.5 \% \mathrm{w} / \mathrm{v})$ for $\sim 15 \mathrm{~min}$.

Biotinamide-filled neurons were intensely labelled with an insoluble, black/blue precipitate. Sections were slide-mounted and coverslipped using DPX mounting media (Sigma-Aldrich). Neurons were traced in three dimensions using Neurolucida software (v9.0; MBF Bioscience, Williston, VT, USA) and a light microscope (Nikon Eclipse $80 \mathrm{i}$ ) at $63 \times$ magnification, with a motorised $\mathrm{x}-\mathrm{y}-\mathrm{z}$ stage and digital camera (MBF Bioscience).

This article is protected by copyright. All rights reserved. 
In vitro unit recordings of nucleus incertus neurons and response to CRF

Rats (dams and pups) were housed in a standard animal facility with food and water available ad libitum and constant environmental conditions $\left(22-23^{\circ} \mathrm{C}, 12\right.$ hour light/dark cycle, lights on at 8:00 a.m.). Young male Wistar rats (2-3 weeks old) used for brain slice preparations and electrophysiological recordings were deeply anaesthetised with isoflurane, decapitated, and whole brains were dissected and submerged in ice-cold ACSF containing $(\mathrm{mM}): \mathrm{NaCl}$, 118; $\mathrm{NaHCO}_{3}, 25 ; \mathrm{KCl}, 3 ; \mathrm{NaH}_{2} \mathrm{PO}_{4}, 1.2 ; \mathrm{CaCl}_{2}, 2 ; \mathrm{MgCl}_{2}$, 2; and glucose, 10; $\mathrm{pH}$ 7.4; osmolarity 290-300 mosmol/kg. Coronal slices $(250 \mu \mathrm{m})$ containing the NI were cut on a Leica VT1000S microtome (Leica Instruments, Heidelberg, Germany) and transferred to the pre-incubation chamber containing carbogenated, warm $\left(32^{\circ} \mathrm{C}\right)$ ACSF for $60 \mathrm{~min}$. After a $1 \mathrm{~h}$ recovery period individual slices were placed in the submerged recording chamber where the tissue was continuously perfused with carbogenated ACSF $(2.5 \mathrm{ml} / \mathrm{min})$. The recording chamber was placed on a fixed stage of an Axio Skope 2 microscope equipped with videoenhanced infrared-differential interference contrast (Zeiss, Göttingen, Germany).

Whole cell current clamp recordings were performed with pipettes pulled from borosilicate glass capillaries (4-6 M $\Omega$ ) on a horizontal puller (Sutter Instruments, Novato, CA, USA) and filled with (mM): potassium gluconate, $125 ; \mathrm{KCl}, 20 ; \mathrm{MgCl}_{2}, 2 ; \mathrm{Na}_{2} \mathrm{ATP}, 4$; $\mathrm{Na}_{3}$ GTP, 0.4; EGTA, 1; and HEPES, 10; pH 7.3; osmolality 290-300 mosmol/kg. The calculated liquid junction potential using this solution was $14.8 \mathrm{mV}$, and the data were corrected for this offset. Recording pipettes were filled with biocytin ( $0.1 \%$; Sigma-Aldrich, Schnelldorf, Germany) for filling of recorded cells and subsequent immunohistochemical examination. All recordings were performed at $32^{\circ} \mathrm{C}$. Cell attached and subsequent wholecell configurations were obtained using negative pressure delivered through the recording pipette using ez-gSEAL pressure controller (NeoBiosystem, San José, CA, USA). An SEC 05LX amplifier (NPI, Tamm, Germany) and Signal and Spike2 (Cambridge Electronic Design) software were used for data acquisition and further analysis, as well as a custommade script in MATLAB (MathWorks Inc., Natick, MA, USA). The change in recorded signal in response to the drug application was considered significant if it differed from the mean resting potential by more the three standard deviations. The output signal was low-pass filtered at $5 \mathrm{kHz}$ and digitised at $15 \mathrm{kHz}$. Continuously monitored input resistance allowed for elimination from the analysis cells with unstable access resistance.

After electrophysiological recordings, slices were transferred to $4 \% 4 \%$ formaldehyde in PBS (made from paraformadehyde) for 24h. After several washes in PBS, slices were 
incubated 10\% normal donkey serum (Jackson ImmunoResearch, Newmarket, UK) and 0.6\% Triton X-100 (Sigma-Aldrich) in PBS overnight. Sections were incubated for $48 \mathrm{~h}$ with 1:200 dilution of Cy3-conjugated Extravidin (Sigma-Aldrich) and 1:1000 dilution of monoclonal mouse anti-RLN3 (HK4-144-10) with 2\% NGS and 0.1\% Triton X-100 in PBS at $4^{\circ} \mathrm{C}$. After washing, slices were incubated with 1:400 dilution of donkey anti-mouse DyLight649 antibody (Jackson ImmunoResearch) or Alexa-488 (Invitrogen). Sections were mounted on glass slides, coverslipped with Vectashield containing DAPI (Vector Laboratories, Peterborough, UK) and examined using an Axiovert 200 confocal laser microscope (Zeiss, Jena, Germany).

\section{Chemicals and drugs}

For application of CRF in vivo, a trephine hole was drilled in the skull to allow placement of a 30-gauge injector needle filled with a $1 \mu \mathrm{g} / \mu \mathrm{l}$ solution of human/rat CRF (Auspep, Tullamarine, VIC, Australia) diluted in 0.05 M Tris-buffered saline (pH 7.6) into the lateral ventricle ( $20^{\circ}$ angle; AP, $-0.2 \mathrm{~mm}$; ML, $+1.5 \mathrm{~mm}$; DV, $-2 \mathrm{~mm}$ from bregma). Once an NI neuron was identified, a control recording of basal firing $>60 \mathrm{~s}$ was followed by icv infusion of CRF $(0.33,1$ or $3 \mu \mathrm{g}$ doses $)$ and $10 \mathrm{~min}$ recording. All drugs were applied to slices by bath perfusion. CRF, TTX (tetrodotoxin citrate) and NBI35965 hydrochloride were purchased from Tocris Bioscience (Bristol, UK). CRF peptide used in in vivo and in vitro studies from Auspep and Tocris Bioscience, respectively, were of $\geq 95 \%$ purity and identical peptide sequence: Ser-Glu-Glu-Pro-Pro-Ile-Ser-Leu-Asp-Leu-Thr-Phe-His-Leu-Leu-Arg-Glu-ValLeu-Glu-Met-Ala-Arg-Ala-Glu-Gln-Leu-Ala-Gln-Gln-Ala-His-Ser-Asn-Arg-Lys-Leu-MetGlu-Ile-Ile- $\mathrm{NH}_{2}$, analogous to human and rat CRF.

\section{Immunohistochemical staining for $C R F-R 1$ and relaxin-3}

Adult male Sprague-Dawley rats (300-350 g; $\mathrm{n}=8$; Australian Research Centre) were used following 7 days acclimatisation to holding facility. All efforts were made to minimise stress prior to experimentation. During the light-phase (12-3 pm), rats were deeply anesthetised with pentobarbitone (100 mg/kg; i.p.) and transcardially perfused with $300 \mathrm{ml}$ of ice-cold 0.1 M phosphate-buffered saline (PBS), pH 7.4 followed by $500 \mathrm{ml}$ of 4\% formaldehyde in PBS (made from paraformadehyde), as described (Ma et al. 2007). Rats were then decapitated, and brains were dissected and submerged in $30 \%$ sucrose in PBS for $48 \mathrm{~h}$ at $4{ }^{\circ} \mathrm{C}$. Coronal sections $(40 \mu \mathrm{m})$ through the entire NI were cut on a cryostat at $-16^{\circ} \mathrm{C}$ (Cryocut 1800 ; Leica 
Microsystems) and collected free-floating into PBS immediately before immunohistochemical processing.

Sections were incubated in blocking buffer (10\% v/v normal goat serum (NGS) in PBS) for $1 \mathrm{~h}$ with agitation at room temperature. Sections from four rats were then incubated in primary antibody mix of 1:1000 dilutions of goat anti-CRF-R1/2 (C-20; Santa Cruz Biotechnology Inc, Santa Cruz, CA, USA; (Chen et al. 2000; Tanaka et al. 2005) and mouse anti-RLN3 (HK4-144-10) (Kizawa et al. 2003; Tanaka et al. 2005), 2\% NGS in PBS containing $0.1 \%$ TritonX-100 overnight at $4^{\circ} \mathrm{C}$. The polyclonal antiserum against CRF-R1/2 was raised against the $\mathrm{C}$-terminus of human CRF-R1 that is conserved in rat and mouse CRF$\mathrm{R} 1$. The specificity of this antibody has been described - preabsorption with the antigenic peptide completely abolished CRF-R1 immunostaining on Western blots and in mouse brain sections and displayed no staining in mouse heart sections, known to express only CRF-R2 (Chen et al. 2000). To assess staining specificity of this antibody in NI neurons, we performed antibody preabsorption combining 1:1000 dilution of the antibody with blocking peptide (C-20P; Santa Cruz Biotechnology Inc.) at 0, 1, 2, 10 and 30× relative concentrations for $48 \mathrm{~h}$ at $4^{\circ} \mathrm{C}$. Coronal sections containing NI (4-5 sections) were incubated in these solutions for a further $48 \mathrm{~h}$ at $4^{\circ} \mathrm{C}$ and processed for RLN3- and CRF-R1/2-like immunostaining (as described below).

The RLN3 antiserum was raised in mouse, so a commonly used mouse neuronal nuclear antigen (NeuN) antibody could not be used to stain neuronal nuclei. Instead, bisbenzimide was used as a nuclear marker. Sections were washed $3 \times 10 \mathrm{~min}$ and incubated in a 1:200 dilution of donkey anti-goat-conjugated Alexa488 IgG (Invitrogen Corporation, Sydney, Australia) with gentle agitation for $1 \mathrm{~h}$ at room temperature, followed by $3 \times 10 \mathrm{~min}$ washes. Sections were then incubated in 1:200 dilution of goat anti-mouse-conjugated Alexa549 secondary IgG (Invitrogen) and 1:2000 dilution bisbenzimide (10 $\mu \mathrm{g} / \mathrm{ml} \mathrm{Hoechst}$ 33342; Sigma-Aldrich, Sydney, Australia) with gentle agitation for $1 \mathrm{~h}$ at room temperature, followed by $3 \times 10$ min washes. Sections were slide mounted in DAKO fluorescent mounting medium (DAKO Australia) and coverslipped.

In a separate study ( $\mathrm{n}=4$ brains), sections were similarly processed with 1:1000 dilution of goat anti-CRF-R1/2 (C-20; Santa Cruz Biotechnology Inc.) and 1:5000 dilution of mouse anti-NeuN IgG (Millipore, Temecula, CA, USA) followed by incubation in 1:200 dilutions of donkey anti-goat conjugated Alexa488 and anti-mouse conjugated Dylight409 IgG (Jackson ImmunoResearch Laboratories). 
Sections were photographed using a Leica DM LB2 microscope (Leica Microsystems) equipped with an Optronics camera (Optronics, Goleta, CA, USA). Every section through the NI was imaged sequentially using $10 \times$ magnification to collect light emitted as red, green or blue fluorescence. Images were adjusted for brightness and contrast using ImageJ (NIH). The borders of the NI were defined medially by the densely packed cells of the pars compacta, laterally by the loosely arranged cells of the pars dissipata, anteriorly by the dorsal raphé nucleus and caudally by the prepositus hypoglossal nucleus, ventral to the dorsal tegmental nucleus (Goto et al. 2001; Olucha-Bordonau et al. 2003). Immunoreactivity was used to manually count the numbers of RLN3 only, CRF-R1 only, RLN3 and CRF-R1, and total cell and neuron populations.

\section{Retrograde tracing from nucleus incertus and immunohistochemical staining for CRF}

Adult male Sprague-Dawley rats (250-350 g; $\mathrm{n}=5$ per tracer; Harlan Iberica, Barcelona, Spain) were anesthetised with ketamine (Imalgene; $55 \mathrm{mg} / \mathrm{kg}$ i.p.; Merial Laboratorios SA, Barcelona Spain) and xylacide (Xilagesic; $20 \mathrm{mg} / \mathrm{kg}$ i.p.; Lab Calier, Barcelona, Spain) and placed into a stereotaxic frame (Kopf Instruments). Trephine holes were drilled in the skull based on stereotaxic coordinates taken from the atlas of Paxinos and Watson (1998). For retrograde tracer experiments, Fluorogold retrograde tracer (FG; 5-hydroxystilbamidine; Biotium, Hayward, CA, USA), diluted $4 \%$ in distilled water, was pressure injected to deliver 5-20 nl using an IM-300 microinjector (Narishige, Tokyo, Japan) attached to a $40 \mu \mathrm{m} \mathrm{I.D.}$ glass micropipette lowered into the NI (AP, -9.6 mm; ML, $0.0 \mathrm{~mm}$; DV, -7.4 mm from bregma). A $26 \mathrm{G}$ cannula was implanted in the lateral ventricle (AP, $-0.9 \mathrm{~mm}$; ML, $-1.3 \mathrm{~mm}$; DV, $-3.8 \mathrm{~mm}$ from bregma) with a jeweller's screw and dental cement. Rats were then sutured and allowed to recover for 6 days. In an effort to maximise the visualisation of CRF neuronal populations, rats were re-anesthetised with isoflurane on day 7 post-surgery and injected with $60 \mu \mathrm{g}$ colchicine in ACSF (Sigma-Aldrich) via insertion of a 30G injector needle into the cannula, which was connected to a $5 \mu$ Hamilton syringe (Harvard Apparatus, Holliston, MA, USA) with polyethylene tubing. Rats were allowed to survive for a further 24 h.

Rats were then deeply anesthetised with Nembutal (150 mg/kg i.p.; Euthalender, Barcelona, Spain) and transcardially-perfused with saline $(250 \mathrm{ml})$ followed by $4 \%$ formaldehyde in 0.1 M PB (made from paraformadehyde; Sigma-Aldrich), $\mathrm{pH} 7.4$ for $30 \mathrm{~min}$ $(\sim 500 \mathrm{ml})$. Brains were isolated from the skull and immersed in the same fixative overnight at $4^{\circ} \mathrm{C}$. They were then transferred to $30 \%$ sucrose in PBS for $48 \mathrm{~h}$ at $4^{\circ} \mathrm{C}$. Brains were 
blocked at the level of the flocculi using a rat brain methacrylate matrix (World Precision Instruments, Sarasota, FL, USA) to obtain reliable sections of equal orientation from each rat. Coronal sections $(40 \mu \mathrm{m})$ through the entire brain were collected free-floating into PBS using a freezing slide microtome (Leica SM2010R, Leica Microsystems, Heidelberg, Germany). For each brain, 6 series of sections were obtained.

For permanent double-labelling of retrogradely-transported FG and CRF, a combination of diaminobenzidine (DAB) brown staining for CRF and tetramethyl benzidineammonium heptamolybdate (TMB-AHM) green staining for FG was used. The latter is a modification of a previously used protocol (Olucha et al. 1985). Sections were rinsed $3 \times 5$ min in Tris-buffered saline (TBS), pH 7.6 and incubated in blocking buffer ( $2 \%$ bovine serum albumin and $4 \%$ normal donkey serum in TBS containing $0.01 \%$ Triton X-100) for 1 h. Sections were transferred to primary antibody solution containing 1:2000 dilution of rabbit anti-CRF IgG (Peninsula laboratories, San Carlos, CA, USA) in blocking buffer for $48 \mathrm{~h}$ at $4^{\circ} \mathrm{C}$. Sections were washed $3 \times 5 \mathrm{~min}$ in TBS and incubated in 1:200 dilution of biotinylated donkey anti-rabbit IgG (Jackson ImmunoResearch Laboratories) for $1 \mathrm{~h}$. Following $3 \times 5 \mathrm{~min}$ washes in TBS, Sections were incubated in avidin-biotin complex solution (ABC; VectaStain Elite; Vector Laboratories). Immunostaining of CRF was visualised using standard peroxidase-chromogen brown reaction by placing the sections in $0.25 \mathrm{mg} / \mathrm{ml} \mathrm{3,3'-}$ diaminobenzidine (DAB; Sigma-Aldrich) with addition of $0.003 \% \mathrm{H}_{2} \mathrm{O}_{2}$ for $20 \mathrm{~min}$. Sections were then rinsed $2 \times 10 \mathrm{~min}$ and $2 \mathrm{~h}$ in TBS and transferred into 1:3000 dilution of rabbit anti-FG IgG in blocking buffer overnight at room temperature. Sections were washed $3 \times 10$ min in TBS and incubated in 1:200 dilution of biotinylated donkey anti-rabbit IgG for 90 min. Sections were then washed $3 \times 10 \mathrm{~min}$ in TBS, incubated in $\mathrm{ABC}$ solution for $10 \mathrm{~min}$ and washed $2 \times 5 \mathrm{~min}$ in TBS. Immunostaining of FG was visualised as a dark blue reaction product by incubating the sections in $0.25 \%$ ammonium heptamolybdate (Merck) in $0.05 \mathrm{M}$ acetate buffer, $\mathrm{pH} 5$ for $30 \mathrm{~min}$. Sections were then transferred into a solution containing 2 $\mathrm{mg} / \mathrm{ml}$ tetramethylbenzidine dissolved in absolute ethanol (Sigma-Aldrich), $100 \mathrm{mg} / \mathrm{ml}$ of ammonium heptamolybdate (Panreac, Barcelona, Spain) and $0.003 \% \mathrm{H}_{2} \mathrm{O}_{2}$ in acetate buffer $(0.5 \mathrm{M}, \mathrm{pH} 5.0)$ for $20 \mathrm{~min}$. Sections were washed $3 \times 10 \mathrm{~min}$ in acetate buffer and mounted onto gelatine-chrome alum-coated glass slides. Mounted sections were air-dried, dehydrated in ascending series of ethanol, cleared in xylene and coverslipped with DPX mounting medium (Sigma-Aldrich). 
Data analysis

All data are expressed as mean \pm SEM. Comparison of data was performed using either ANOVA followed by a Bonferroni post hoc multiple comparisons test for analysis of multiple groups of data or paired or unpaired $t$-test. All tests were two-tailed. Results were considered to be statistically significant if $P<0.05$.

\section{Results}

\section{Firing characteristics of NI neurons and their response to CRF in vivo and in vitro}

Electrophysiological properties of NI neurons and response to CRF in vivo

In a series of studies, we assessed the electrophysiological characteristics of NI neurons in vivo in combination with juxtacellular labelling to assess the presence or absence of RLN3-IR in recorded neurons. The locations of all juxtacellularly-labelled and characterised neurons within the NI are illustrated in Figure 1.

Initial experiments using isoflurane anaesthesia during recording revealed a large proportion of silent NI neurons, whereas all neurons were spontaneously firing under urethane anaesthesia. Therefore, only results from recordings under urethane anaesthesia were analysed. In our hands, arterial pressure was generally unaltered or increased up to 15 $\mathrm{mm}$ Hg following icv CRF infusion (see Figs. $2 A$ and $3 A$, respectively, for examples). In 47 rats, a total of 63 NI neurons were recorded. Preliminary recordings tested CRF at $0.3,1$ and $3 \mu \mathrm{g}$ doses. Compared to basal firing $(3.4 \pm 1.1 \mathrm{~Hz}, \mathrm{n}=28)$, all doses of CRF resulted in significant activation of spontaneous firing in some cells $(0.3 \mu \mathrm{g}$ : $7.0 \pm 1.7 \mathrm{~Hz}, \mathrm{n}=10 ; 1 \mu \mathrm{g}$ : $\left.8.5 \pm 1.7 \mathrm{~Hz}, \mathrm{n}=10 ; 3 \mu \mathrm{g}: 7.5 \pm 2.4 \mathrm{~Hz}, \mathrm{n}=18 ; F_{(3,55)}=8.34, P<0.01\right)$. Inhibition of spontaneous firing of some cells was also observed at all 3 doses, however only the $3 \mu \mathrm{g}$ CRF dose produced a statistically significant decrease below basal firing $(1.3 \pm 0.4 \mathrm{~Hz}, \mathrm{n}=18$; $\left.F_{(3,56)}=3.41, P<0.05\right)$ compared to $0.3 \mu \mathrm{g}$ CRF $(2.4 \pm 0.8 \mathrm{~Hz}, \mathrm{n}=8)$ or $1 \mu \mathrm{g}$ CRF $(1.8 \pm 0.7$ $\mathrm{Hz}, \mathrm{n}=8$ ). Hence, the $3 \mu \mathrm{g}$ dose was used in the majority of experiments to assess CRF activation and inhibition of NI neurons ( $n=38$ rats, $n=43$ neurons).

Of the 43 neurons assessed with $3 \mu \mathrm{g}$ CRF, 20 were antidromically-activated from the medial septum and the existence of an axonal projection to the medial septum was confirmed in each via a positive collision test (see Fig. $2 C$ for example). Fourteen neurons were orthodromically driven by afferent projections from the medial septum (see Fig. $3 C$ for example) and 8 neurons were neither antidromically nor orthodromically activated, despite 
being spontaneously active. Neurons were categorised based on their response to CRF as 'activated', 'inhibited' or 'unaffected'. Approximately 50\% of NI neurons were successfully juxtacellularly-labelled. The heterogenous responses and firing characteristics of these neurons are summarised in Figure 4. No significant differences were detected between labelled and unlabelled neurons in all parameters measure $(P=0.9$, one way ANOVA) and therefore data was pooled for each group.

Following icv infusion of CRF, the firing of 18 neurons was significantly increased compared to baseline firing $(7.5 \pm 2.4$ and $3.4 \pm 1.7 \mathrm{~Hz}$, respectively; $P<0.001$; Fig. $4 A)$. The time from infusion to onset of CRF effect on neuronal firing was $170 \pm 35.3 \mathrm{~s}(\mathrm{n}=18$; Fig. $4 C$ ). Of the 18 neurons, 9 neurons were antidromic and 6 neurons were orthodromic to stimulation of the medial septum. A previous study of NI neurons described 2 types rhythmic (type I) and non-rhythmic (type II) firing cells (Nunez et al. 2006). The majority of NI neurons activated by CRF were non-rhythmic (15 neurons), but 3 neurons were classified as rhythmic. Ten of the 18 neurons were successfully juxtacellularly-labelled - 9 were confirmed to be RLN3-positive and 1 neuron was confirmed RLN3-negative (Table 1). An example of the firing response to CRF of an antidromic, RLN3-positive neuron is illustrated (Fig. 2). Of the RLN3-positive neurons activated by CRF, 5 were antidromic (17.2 $\pm 2.9 \mathrm{~ms}$ delay) and 3 were orthodromic $(20.3 \pm 6.6 \mathrm{~ms}$ delay) to stimulation of the medial septum (Table 1), and 8 were classified as non-rhythmic and 1 as rhythmic firing. Average spike duration (peak depolarisation to hyperpolarisation) of neurons activated by CRF was $0.57 \pm$ $0.05 \mathrm{~ms}$, which was not significantly different from the equivalent value for those confirmed RLN3-positive $(0.66 \pm 0.9 \mathrm{~ms} ; P=0.4)$ and that of the confirmed RLN3-negative neuron $(0.53 \mathrm{~ms})$. Furthermore, spike duration of CRF-activated neurons did not differ significantly between those that were antidromic $(0.69 \pm 0.1 \mathrm{~ms})$ or orthodromic $(0.45 \pm 0.5)$ to stimulation of the medial septum $(P=0.12)$.

Following juxtacellular-labelling with biotinamide, neurons were digitally reconstructed to assess neuronal morphology. A representative tracing of an RLN3 neuron activated by CRF is illustrated in Fig. $2 E$. These neurons are typically multipolar, had an elongated cell body with 2-5 main dendritic trees of long, aspiny dendrites that were sparsely branching and restricted within the NI. Each neuron consisted of a large axon that generally traversed laterally and/or rostrally, with little to no branching within the NI. Many of these neurons were confirmed antidromically-stimulated by medial septum stimulation and have been previously shown to have projections to many other distant forebrain regions (Goto et 
al. 2001; Olucha-Bordonau et al. 2003), thus it is likely that these neurons send long axons to distant target regions and full recovery would be technically difficult.

Following icv infusion of CRF, the firing of 19 neurons was significantly decreased $(2.2 \pm 1.0 \mathrm{~Hz})$ compared to baseline firing $(4.6 \pm 1.1 \mathrm{~Hz} ; P<0.001 ;$ Fig. $4 A)$. Time from infusion to onset of CRF effect on neuronal firing was $114.0 \pm 25.5 \mathrm{~s}$ (Fig. 4C). Of the 19 neurons, 6 neurons were antidromic and 9 neurons were orthodromic to stimulation of the medial septum. The majority of NI neurons inhibited by CRF were non-rhythmic $(n=11)$, but 8 neurons were classified as rhythmic, a greater number compared to CRF activated neurons. Juxtacellular labelling was successfully achieved in 8 of the 19 neurons and all labelled neurons were confirmed RLN3-negative (Table 1). An example of the firing response to CRF of an orthodromic, RLN3-negative neuron is illustrated (Fig. 3). Of the 8 labelled neurons, 1 neuron was antidromic ( $25 \mathrm{~ms}$ delay) and 5 were orthodromic (39.45 \pm $17.5 \mathrm{~ms}$ delay) to stimulation of the medial septum, and 5 were classified non-rhythmic and 3 as rhythmic firing (Table 1). Average spike duration of neurons inhibited by CRF was $0.44 \pm$ $0.04 \mathrm{~ms}$, and those confirmed as RLN3-negative were not significantly different $(0.41 \pm 0.05$ $\mathrm{ms}$ ). An example of a non-RLN3 CRF-inhibited neuron is illustrated in Fig. $3 E$. In this case, the stellate-shaped neuron consisted of five long, aspiny dendrites that were sparsely branching within the NI and a large axon that traversed rostrally.

The firing of a small group of neurons recorded (6 of 43) were unaffected by CRF. Average firing rate of these neurons was $4.3 \pm 1.4 \mathrm{~Hz}$ (Fig. 4B) and they consisted of rhythmic $(\mathrm{n}=3)$ and non-rhythmic $(\mathrm{n}=3)$ neurons. Average spike duration of neurons unaffected by CRF was $0.44 \pm 0.07 \mathrm{~ms}$. All 4 labelled neurons were confirmed negative for RLN3-IR (Table 1).

A comparison of the neurons that displayed the three different responses to CRF revealed no significant difference in basal firing rates between CRF activated $(3.4 \pm 1.7 \mathrm{~Hz})$, inhibited $(4.6 \pm 1.1 \mathrm{~Hz})$ and unaffected units $\left(4.3 \pm 1.4 \mathrm{~Hz} ; F_{(2,36)}=2.49 ; P=0.15\right.$; Fig. $\left.4 B\right)$. The firing frequencies of neurons activated by CRF infusion $(7.5 \pm 2.4 \mathrm{~Hz})$ was significantly different from those that were inhibited $(2.2 \pm 4.2 \mathrm{~Hz} ; P<0.05)$. There was no significant difference in the time to onset of CRF effects between activated (170 $\pm 35.3 \mathrm{~s})$ and inhibited neurons (114 $\pm 25.5 \mathrm{~s} ; P=0.2$; Fig. $4 C)$. Furthermore, there was no significant difference in the spike duration of neurons across the 3 groups $(P=0.22)$. Our in vivo studies however, did not examine whether the observed effects of CRF on firing were pre- or post-synaptic or confirm the specificity of effects to CRF-R1. These issues were addressed in electrophysiological recordings in vitro. 
Electrophysiological responses of NI neurons to CRF in vitro

In studies to further examine whether CRF effects on NI neuron firing were direct, we made recordings of NI neurons in an in vitro coronal brain slice preparation. This allowed us to evaluate the electrophysiology of these neurons free of synaptic inputs that may account for some of the effects of CRF observed in vivo. The cerebrospinal fluid volume in the adult rat brain is 100-150 $\mu \mathrm{l}$ (Davson 1969) and therefore the $3 \mu \mathrm{g}$ dose of CRF administered icv is equivalent to 4-7 $\mu \mathrm{M}$, if evenly distributed throughout the ventricular system. Because of significantly less buffering in the slice preparation, we tested CRF doses in vitro below those used for in vivo studies.

The majority of in vitro recordings were made from neurons in NI pars compacta, where the boundaries of the nucleus in the slice are relatively clear. Bath application of 100 nM CRF affected 79\% of recorded neurons (15 of 19 neurons; see example in Fig. 5A). Ten of 15 CRF activated neurons recorded in standard ACSF were successfully labelled with biocytin, and the mean recorded increase in firing rate of these neurons in response to CRF was $1.6 \pm 0.4 \mathrm{~Hz}$. The mean baseline firing frequency of activated cells was $1.8 \pm 0.6 \mathrm{~Hz}$, calculated from $300 \mathrm{~s}$ of stable activity before CRF application, and there were no significant differences in baseline firing frequency between RLN3-positive $(n=5)$ and RLN3-negative $(\mathrm{n}=5)$ neurons $(2.1 \pm 1.1 \mathrm{~Hz}$ and $1.5 \pm 0.6 \mathrm{~Hz} ; P=0.7)$ or magnitude of mean spike firing frequency increase in response to $100 \mathrm{nM} \mathrm{CRF}(2.4 \pm 0.7 \mathrm{~Hz}$ and $1.2 \pm 0.4$ in RLN3-positive and RLN-negative cells, respectively; $P=0.2$ ). Four recorded and labelled NI cells were unaffected by CRF and these cells were confirmed RLN3-negative (data not shown). In neurons that were activated by $\mathrm{CRF}$, spike shape analysis revealed significant reductions in the afterhyperpolarisation (AHP) peak, from $-73.1 \pm 0.9 \mathrm{mV}$ at baseline to $-67.7 \pm 2 \mathrm{mV}$ after CRF treatment (mean calculated from an average of 50 spikes; $P=0.02$ ) and AHP duration was $222 \pm 24 \mathrm{~ms}$ at baseline and $143 \pm 23 \mathrm{~ms}$ after CRF treatment (mean calculated from an average of 50 spikes, taken as a period between the minimum peak in hyperpolarisation to the baseline potential level; $P=0.02$; Fig. $5 B$ ). Spike shape measurements were taken from the actual membrane potential before and during CRF, respectively. Various doses of CRF (10, $30,100,300,600 \mathrm{nM}$ ) were tested to assess dose-dependent changes in CRF effects. At all doses, CRF depolarised NI neurons and the magnitude of membrane potential change was dose-dependent (Fig. 5C). To assess whether CRF receptors display desensitisation, we recorded the activity of NI neurons to multiple, consecutive applications of $100 \mathrm{nM} \mathrm{CRF}$ and 
observed consistent depolarisation, suggesting that the actions of CRF did not lead to CRF receptor desensitisation (Fig. 5D).

In studies to determine whether CRF directly excited recorded neurons, 7 neurons that were activated by $100 \mathrm{nM}$ CRF were further tested for CRF response in the presence of TTX $(1 \mu \mathrm{M}$; Fig. $5 E)$. All neurons tested in the presence of TTX were depolarised by CRF $(3.1 \pm$ $0.3 \mathrm{mV}$ ). The mean baseline membrane potential of all neurons depolarised by CRF was $66.1 \pm 3.3 \mathrm{mV}$. After establishing that CRF directly activated NI neurons, we tested effects of the CRF-R1 antagonist NBI $35965(1 \mu \mathrm{M})$ on the resting activity of NI neurons. Neither the frequency of spike firing nor resting membrane properties were altered in the presence of NBI 35965, suggesting there is little to no CRF-R1-mediated tone in the NI brain slice preparation ( $\mathrm{n}=3$; data not shown). Neurons activated by $100 \mathrm{nM} \mathrm{CRF}$ (in the presence of TTX) were subsequently treated with NBI $35965(1 \mu \mathrm{M})$ for $5 \mathrm{~min}$ before, during and for 5 min after a second CRF application $(n=3$; Fig. $5 E)$. All neurons failed to respond to CRF reapplication, supporting a CRF-R1-mediated action of CRF in the NI.

Of 6 neurons successfully labelled, 4 were confirmed to be RLN-positive (see example Fig. $5 F$ ) and 2 were RLN3-negative. In cases where neurons were well labelled, we observed similar neuron morphology to that observed following juxtacellular-labelling in vivo. An example of an RLN3 neuron is illustrated in Fig. $5 G$ showing long, aspiny dendrites and initial axon observed in the $250 \mu \mathrm{m}$ slice.

Changes in the input resistance of the recorded neurons were monitored with hyperpolarizing current pulse injections (50 pA, $1.5 \mathrm{~s}$ duration). In neurons activated by CRF, with depolarisation of membrane potential in the presence of TTX, a small decrease in input resistance from $422.6 \pm 53.8 \mathrm{M} \Omega$ to $396.4 \pm 47 \mathrm{M} \Omega(P=0.04)$ was observed (data not shown).

\section{Coherence analysis of NI neuron firing with hippocampal EEG}

The integrity of the NI has been demonstrated to be important in the brainstem generation of hippocampal theta rhythm (Nunez et al. 2006); and as local field potentials of the NI and hippocampus show strong coherence at theta frequency (Cervera-Ferri et al. 2011), we assessed the coherence of firing of NI neurons during hippocampal theta epochs. From 28 urethane-anesthetised rats, we recorded the spontaneous firing of NI neurons in 2 different states: one associated with large-amplitude irregular activity in the hippocampus and the other characterised by the presence of sustained, rhythmic theta oscillations $\sim 4 \mathrm{~Hz}$. Mean firing frequency of RLN3 neurons during theta epochs was $7.84 \pm 4.45 \mathrm{~Hz}(\mathrm{n}=6)$, where 5 
neurons fired between $3.4-4.6 \mathrm{~Hz}$ (Fig. $6 B$ ) and 1 neuron showed strong, rhythmic burst firing at $30 \mathrm{~Hz}$ (Fig. 6C). As this neuron were confirmed RLN3-positive, it was included in further analyses. The firing of all RLN3 neurons was consistently and strongly modulated with the initial ascending phase of hippocampal theta oscillations (Fig. 6A and $B$ ). Mean angle was $91.3^{\circ}$ (range $53.8^{\circ}-124.9^{\circ}$ ) and mean $r$ was 0.49 (Fig. 6C). As a population, RLN3 neurons were found to have tightly synchronised firing with theta oscillations $\left(\mathrm{R}^{\prime}=\right.$ $1.12, \mathrm{R}_{0.05,6}=1.074$, Moore's test).

Mean firing frequency of non-RLN3 neurons during theta epochs was $5.31 \pm 0.34 \mathrm{~Hz}$ (range $4.2-6.1 \mathrm{~Hz} ; \mathrm{n}=6$ ) and had varied firing coherence during theta oscillations between $144^{\circ}-324^{\circ}$ (Fig. $6 A$ and $D$, mean $\mathrm{r}=0.14$ ). One neuron showed some coherent firing with the descending phase of theta oscillations (mean angle $=320.7^{\circ}, \mathrm{r}=0.58$ ). Therefore, this population showed no statistically phase-locked firing modulation with hippocampal theta oscillations $\left(\mathrm{R}^{\prime}=0.49, \mathrm{R}_{0.05,6}=1.074\right.$, Moore's test).

Together, these results suggest that the firing of RLN3 neurons is strongly modulated and phase-locked to hippocampal theta oscillations, where in some cases neurons show rhythmic, burst-type firing. In contrast, non-RLN3 neurons show little phase-locked firing with theta oscillations.

\section{Relaxin-3 neurons in NI express CRF-R1, but not all CRF-R1 positive cells contain relaxin-3}

Previous studies of CRF-R1 expression in the NI reported a very 'high density' of CRF-R1 mRNA, but noted a lack of CRF-R2 mRNA (Bittencourt \& Sawchenko 2000; Van Pett et al. 2000; Justice et al. 2008). More recently, it was reported that 'almost all' RLN3 neurons of the rat NI co-expressed CRF-R1 immunoreactivity (Tanaka et al. 2005). Therefore, using the same antisera as Tanaka and colleagues, we performed a quantitative study of CRF-R1 and RLN3 immunoreactivity (-IR) in relation to the total NI cell population to more thoroughly evaluate their relative distributions in this nucleus. High levels of CRF-R1-and RLN3-IR were observed throughout the NI (Fig. 7A and $B$ ). CRF-R1-IR was finely punctate and outlined neuronal soma, consistent with localisation of the receptor on cell membranes (Fig. $7 B$; Chen et al. 2000), in contrast to primarily cytoplasmic staining of cell bodies with RLN3 (Fig. 7C; Tanaka et al. 2005). There was clear evidence of neurons within the NI that were positive and negative for CRF-R1-IR. CRF-R1 positive neurons were observed evenly distributed throughout the compacta and dissipata regions, whereas RLN3-positive neurons were observed predominantly in the compacta and less so in the dissipata. 
In double-label experiments detecting RLN3, we used bisbenzimide as a marker of total cell number, inclusive of neurons and glia (see Methods). Quantification of the left half of the NI of each rat revealed that each $40 \mu \mathrm{m}$ section contained an average of $470 \pm 33$ cells, with the most rostral and caudal sections, caudal to the dorsal raphé, and anterior of the prepositus nucleus, respectively, containing fewer cells (450-550 cells) than mid-NI sections (750-820 cells). In all sections analysed, RLN3-IR co-localised with CRF-R1-IR, though a population of CRF-R1 neurons were negative for RLN3-IR (Fig. 7C-E). The mean number of CRF-R1 neurons per hemi NI was $53.6 \pm 2.9$, equivalent to $11.4 \%$ of the total cell population. Of the CRF-R1 neurons, $28.4 \pm 2.8$ were immune-positive for RLN3, equating to $53 \%$ of the CRFR1 population.

In a separate experiment in which sections were processed for CRF-R1 - and NeuN-IR, both colocalised in all sections analysed (Fig. $7 F-H$ ). The mean number of neurons per hemi NI section was $102.3 \pm 6.7$, of which $51.8 \pm 3.9$ were CRF-R1-IR, indicating that CRF-R1 neurons accounted for $51.9 \pm 3.9 \%$ of the total NI neuronal population. Thus overall, $\sim 53 \%$ of CRF-R1 neurons synthesise RLN3, and CRF-R1 and CRF-R1/RLN3 neurons account for $\sim 52 \%$ and $\sim 28 \%$ of all NI neurons, respectively; whereas $48 \%$ of NI neurons were apparently devoid of both RLN3 and CRF-R1. These data suggest most, if not all, NI RLN3 neurons would be responsive to CRF and a separate, equal sized population of CRF-responsive neurons do not contain RLN3, in addition to an almost equivalent population of NI neurons that do not bear CRF-R1 and would be predicted to be unresponsive to direct actions of CRF.

As part of these studies, we addressed the specificity of the CRF-R1 antisera used, as recently it was reported that it produced 'non-specific' staining in paraformaldehyde-fixed sections of mouse brain (Refojo et al. 2011). Importantly, however, these authors report that the C-20 polyclonal antisera recognises the CRF-R1 in vitro and it should not be assumed that it is broadly non-specific in non-mouse tissues. Preabsorption of the C-20 CRF-R1 antisera, relative to staining in the absence of blocking peptide, at $1 \times$ and $2 \times$ concentrations of blocking peptide resulted in a small, but detectable decrease in bright, punctate-staining (data not shown). The $10 \times$ and $30 \times$ concentrations of blocking peptide resulted in marked and selective decrease in CRF-R1-IR (Supplemental Fig. $1 B$ and $C$ ), where $30 \times$ blocking peptide preabsorption resulted in weak or no immunostaining. Preabsorption had no detectable effect on concurrent RLN3-IR (Supplemental Fig. 1D-F). These findings support the specificity of CRF-R1-IR detected in the NI. However, as there is plasticity in CRF-R1 and RLN3 expression levels, particularly in response to stress (Huang et al. 2006; Banerjee et 
al. 2010), the current immunohistochemical data should be interpreted as a reflection of the heterogeneity of CRF-R1- and RLN3-like immunostaining in the NI of naïve, unstressed, male rats. Importantly though, this conclusion is consistent with the heterogeneous responses to CRF observed in the electrophysiological studies using similar 'unstressed' rats.

\section{Possible forebrain sources of a CRF-containing innervation of NI}

Intracerebroventricular (icv) injection of CRF strongly activates c-fos gene and Fos protein expression in neurons of the NI, which in rats lies adjacent to the $4^{\text {th }}$ ventricle, suggesting the NI may be activated via volume transmission by endogenous CRF present in the cerebrospinal fluid (Bittencourt \& Sawchenko 2000). While the largest population of CRF neurons resides in the hypothalamic paraventricular nucleus and supplies CRF to the median eminence and anterior pituitary (Sawchenko \& Swanson 1985), several other populations of CRF neurons are present in hypothalamus and extra-hypothalamic areas, including the bed nucleus of the stria terminalis (BNST), central amygdala, locus coeruleus, cerebral cortex, cerebellum and spinal cord (Sawchenko \& Swanson 1985) and could provide a direct CRFcontaining innervation of the NI.

In an experiment to ascertain possible forebrain sources of an endogenous CRF innervation of the NI, we injected the retrograde tracer, FG, into the NI and assessed the presence of double-labelling for FG and CRF in relevant areas of the forebrain. Twenty-four hours prior to fixative perfusion, rats were treated with colchicine, icv, to improve the visualisation of CRF-IR in neuronal cell bodies. At the injection sites, FG generally remained confined to the NI region with little spread to the dorsal tegmental nuclei and reticular formation below the medial longitudinal fascicle (Fig. 8B), and there was no spread of tracer to the neighbouring dorsal raphé nucleus. Retrograde-labelling was observed in neurons in the supramammillary nucleus, lateral hypothalamic area, the hypothalamic perifornical area, paraventricular and periventricular nuclei (data not shown) and lateral preoptic area (Fig. 8A), which extended rostrally into the horizontal medial septum. Medium to strong somatic CRFIR was observed in the parvocellular division of the paraventricular hypothalamic nucleus, the medial division of the central amygdala, lateral nuclei of the BNST, median raphé and interpeduncular nuclei (data not shown) and preoptic hypothalamus (Fig. 8C). Co-localisation of FG and CRF-IR was observed in a diffuse area of the lateral preoptic area (LPO, Fig. 8C), although notably, no co-localisation was observed in the paraventricular hypothalamus, central amygdala or BNST. These data suggest that LPO, but not paraventricular hypothalamus, amygdala or BNST, may provide a CRF innervation of the NI. 


\section{Discussion}

$\mathrm{CRF}$ is a primary stress peptide/hormone that is rapidly up-regulated and released from neural populations to mediate adaptive autonomic and behavioural responses to stress (Koob \& Bloom 1985; Aguilera \& Liu 2012) and the neural circuitries underlying these effects are still being identified and defined. In this study, we have characterised a novel brainstem site, the NI, containing a large population of CRF-responsive, CRF-R1-containing GABAergic neurons. We have, for the first time, documented firing properties of NI neurons in vivo and in vitro, their response to CRF and whether the recorded neurons contained the neuropeptide, RLN3. Our findings reveal that the NI is a heterogeneous neuronal population and the majority of neurons activated by CRF contain RLN3.

\section{Direct effects of CRF on NI neurons via CRF-R1}

Previous studies have reported high densities of CRF-R1 and lack of CRF-R2 mRNA in the NI (Bittencourt \& Sawchenko 2000; Van Pett et al. 2000; Justice et al. 2008). We observed CRF-R1-IR that was consistent with postsynaptic, membrane localisation on neuronal soma. The time course of CRF action in activating NI neurons in vivo and in vitro, $\sim 170 \mathrm{~s}$ and $50 \mathrm{~s}$ respectively, is consistent with the nature of CRF-R1 signalling (Punn et al. 2006) and may reflect the speed at which CRF infusion into the lateral ventricle diffuses to NI neurons adjacent to the $4^{\text {th }}$ ventricle in vivo, versus lack of buffering in a slice preparation perfused with CRF in the bath. Furthermore, our in vitro findings demonstrated that CRF activation of NI and RLN3 neurons is dependent on TTX-resistant CRF-R1 binding and activation, consistent with direct postsynaptic effects at CRF-R1.

We also observed a neuronal population that did not express CRF-R1 or contain RLN3 and these were generally inhibited or unaffected by exogenous CRF in vivo. Notably, an equivalent population was not observed in vitro, suggesting that there may be distant extrinsic inhibitory inputs to these neurons that are enhanced following icv CRF administration, but are disconnected in the slice preparation (Fig. 9). The neighbouring serotonergic dorsal raphé (DR) could be a potential inhibitory input, as 5-HT neurons and their fibres intermingle with RLN3/NI neurons that express $5 \mathrm{HT}_{1 \mathrm{~A}}$ receptors (Miyamoto et al. 2008). Furthermore, CRF has been demonstrated to have differential direct and indirect effects on 5-HT and non-5-HT (GABAergic) neurons of the DR in vitro, mediated by CRFR1, CRF-R2 and GABAergic synaptic activity (Kirby et al. 2008). In contrast to the DR study, we did not observe a dose-related differentiation in firing, nor did we observe any 
TTX-sensitive CRF effects. The neurochemical identity of CRF-inhibited, RLN3-negative NI neurons is currently unknown.

The small, but significant, decrease in the input resistance elicited by CRF observed in our in vitro studies of NI neurons may be related to the opening of L-type or other types of calcium channels, as described elsewhere (Yu \& Shinnick-Gallagher 1998; Young et al. 2001). However, as a decrease in input resistance is consistent with the possible opening of an ion channel(s), the closing of a potassium channel, or a combination of both mechanisms, further studies are required to clarify this action.

In some of our in vivo and in vitro experiments, we obtained well-filled neurons that allowed assessment of neuronal morphology. Generally, RLN3 neurons had elongated cell bodies with 2-5 aspiny dendritic trees that extended within the boundaries of the NI (e.g. Figure $2 E$ ). However, greater heterogeneity in morphology was observed of non-RLN3 neurons, which consisted of stellate- (e.g. Figure $3 E$ ) and elongated-shaped neurons. These non-RLN3 neurons may consist of local interneurons, and other GABAergic and glutamatergic neurons that project to the forebrain (Cervera-Ferri et al. 2012), and future studies will fully characterise the neurochemical heterogeneity of NI neurons and their projection targets.

Although the in vitro studies employed young Wistar rats and the in vivo studies used adult Sprague-Dawley rats, previous anatomical and electrophysiological characterisation of the NI suggests that the connectivity and electrophysiological properties of NI neurons do not differ between the Wistar and Sprague-Dawley strains (Nunez et al. 2006). Furthermore, an earlier study of the developmental profile of RLN3/NI neurons indicates that RLN3 mRNA is present in the rat NI from embryonic day 18 and levels are equivalent to those observed in the adult by postnatal day 7 (Miyamoto et al. 2008), suggesting that the NI and RLN3 system in postnatal day 21 rats used for in vitro studies are established and comparable to that of the adult and that the differences observed are not age or strain-based.

\section{LPO CRF neurons innervate the NI}

We identified LPO CRF neurons as a potential source of a CRF projection to the NI, although volume transmission cannot be excluded based on previous findings (Bittencourt \& Sawchenko 2000; Tanaka et al. 2005) and the present study. Earlier studies identified that CRF icv infusion in rats activated NI neurons and Bittencourt and Sawchenko (2000) postulated that with its close proximity to the 4th ventricle, the NI may normally be influenced by CRF in the CSF via volume transmission. In terms of neural CRF sources, the 
largest population of neurons reside in the paraventricular hypothalamic nucleus, although CRF is also synthesised in other limbic brain structures, including the BNST, central amygdala, and in Barrington's nucleus, cerebral cortex, cerebellum, and spinal cord (Sawchenko \& Swanson 1985). In a similar fashion to its receptors, CRF gene expression and mature peptide levels are regulated in several brain regions in response to stressors, such as restraint stress (Kalin et al. 1994), and palatable (Cottone et al. 2009) or regular food deprivation (Nishiyama et al. 2008). Using retrograde-tracing, we identified CRF neurons in the LPO that projected to the NI, although notably under these experimental conditions, no co-localisation was observed in the paraventricular hypothalamus, central amygdala or BNST. LPO innervation of the NI has been documented using the retrograde tracer cholera toxin B (Goto et al. 2001). The LPO is particularly responsive to acute stress, but unlike other stress centres such as the ventrolateral septum, BNST, PVN, DR, median raphé, locus coeruleus and nucleus of the solitary tract, which display desensitisation (i.e. reduced Fos-IR following 9-days restraint stress), LPO neurons are not desensitised (Stamp \& Herbert 2001). It is unclear, however, whether CRF neurons in the LPO are similarly activated during acute and chronic stress.

In these studies we used colchicine treatment to induce the accumulation of CRF within cell bodies, to enhance the visualisation of the peptide in retrograde tracer positive neural populations, as described (Miklos \& Kovacs 2002; Alon et al. 2009). However, as there is the potential for colchicine to induce 'non-specific' peptide production and resultant staining, further studies are warranted to comprehensively address this question, using additional (more sensitive) CRF antisera or CRF in situ hybridisation or CRF promoter-driven viral constructs to better visualise this and other possible CRF neuron populations that project to the NI.

\section{Functional relationship of the NI and the neuropeptide relaxin-3 - role in hippocampal theta rhythm}

The medial prefrontal cortex (largely prelimbic cortex) is a prominent input to the NI, in addition to the septal region, nucleus of the diagonal band, lateral habenula, lateral hypothalamus, and interpeduncular nucleus (Goto et al. 2001). These multimodal afferents suggest the NI is regulated by, and integrates information, relating to behavioural planning associated with prefrontal cortex, lateral habenula and septohippocampal activity (Goto et al. 2001; Olucha-Bordonau et al. 2003). In turn, this is consistent with a capacity for CRF 
activation of NI neurons to enhance the integration of arousal and behavioural processing during stress.

The septohippocampal system is a primary projection target of the NI, particularly the medial and lateral septum, and ventral hippocampus (Goto et al. 2001; Olucha-Bordonau et al. 2003; Olucha-Bordonau et al. 2012). Initial studies revealed that electrical stimulation of NI neurons induced hippocampal theta activity, and lesion of, or muscimol infusion into the NI abolished reticular pontine oralis (RPO)-induced hippocampal theta activity, suggesting the NI mediates brainstem induction of this oscillatory activity (Nunez et al. 2006). The RPO, which lacks direct projections to the septum or hippocampus, innervates the NI that in turn innervates the primary theta pacemaker region of the medial septum (Teruel-Marti et al. 2008). A recent study identified strong coherence between NI and hippocampal local field potentials, where theta activity of the NI preceded that of the hippocampus (Cervera-Ferri et al. 2011). Furthermore, we have demonstrated that NI- and RLN3-containing afferent fibres form dense plexuses in the medial septum and RLN3 is localised in symmetric terminals that synapse onto hippocampal-projecting, choline acetyltransferase-, neuronal nitric oxide synthase-, calbindin-, calretinin-, and parvalbumin-positive neurons (Olucha-Bordonau et al. 2012). In urethane-anesthetised rats or freely-moving rats, RXFP3 agonist and antagonist infusion into the medial septum induced and impaired hippocampal theta activity induced by RPO stimulation or exploration of a context-rich environment, respectively; and RXFP3 antagonist infusion resulted in a dose-related impairment of spatial working memory (Ma et al. 2009a).

More generally, functional studies suggest that RLN3 is a modulator of stress/arousal, feeding/metabolism, and learning/memory (Smith et al. 2011). In RLN3-knockout mice, circadian hypoactivity during the dark phase (reflected by reduced time spent and distance traveled on voluntary running wheels) was observed, with no overt deficits on other behavioral modalities (Smith et al. 2012). A separate study observed increased entries into open arms of the elevated plus maze and an increased acoustic startle response in RLN3knockout mice (Watanabe et al. 2011). These studies support a modulatory role of RLN3 on anxiety and arousal. In recent studies using viral-mediated RLN3 mRNA knock-down in the rat NI, naïve rats displayed no overt effects on various behavioural modalities (Callander $e t$ al. 2012). These findings suggest that under normal, low stress conditions, endogenous tone of RLN3 may be low and thus, in light of current findings that CRF is an upstream activator of RLN3 neurons, behavioural assessment during high endogenous RLN3 tone induced by 
chronic stress or CRF administration may assist the study of the role of this neuropeptide (Tanaka et al. 2005; Banerjee et al. 2010).

In this study, RLN3 neurons exhibited strongly modulated firing activity that was phase-locked with the initial ascending phase of theta oscillations. One of these neurons also displayed rhythmic, burst-type firing that coincided with oscillation peaks. These RLN3 neurons presumably form part of a ponto-septohippocampal pathway that is involved in the modulation of hippocampal theta rhythm, whereas other populations of RLN3 neurons present in adjacent brainstem sites (pontine raphé, periaqueductal grey and dorsal to lateral substantia nigra) (Tanaka et al. 2005; Ma et al. 2007) may participate in neural networks that do not necessarily directly influence hippocampus. Tract-tracing studies have demonstrated that NI neurons densely innervate other brain regions involved in hippocampal theta rhythmogenesis, including the median raphé, supramammillary nucleus and the hippocampus itself, particularly the ventral hippocampus (Goto et al. 2001; Olucha-Bordonau et al. 2003). Furthermore, these regions are innervated by RLN3-containing efferents and contain RXFP3 (Ma et al. 2007). Therefore, it is likely that many of these RLN3 NI neurons will show phaselocked activity during theta activity, but the precise phase and patterns of activity may vary depending on their brain site target. Alternatively, RLN3 neurons may send highly collaterised projections to all of these pacemaker regions and thus coordinate the ascending ponto-septohippocampal pathway in this way.

The NI has been documented across a range of species, including human (Streeter 1903), as a large neuron cluster lying adjacent to the $4^{\text {th }}$ ventricle in the prepontine hindbrain/midbrain (Ryan et al. 2011). We have previously characterised a ventromedial region of the pontine and medullary central grey of the Macaca fascicularis that represents the primate analog of the NI, which expresses dense RLN3 mRNA and peptide (Ma et al. 2009b). Furthermore, the broad distribution of RLN3-IR axons and terminations is highly similar to that observed in the rat (Tanaka et al. 2005; Ma et al. 2007) and mouse (Smith et al. 2010) suggesting that the physiological functions of this neuropeptide system are conserved among mammalian species.

\section{Conclusion}

This study characterised neurons in the NI, a little-studied but functionally important GABAergic brainstem site containing CRF-R1-positive, CRF-responsive neurons in the rat. A large proportion of these activated neurons contain the neuropeptide RLN3 and project broadly throughout the brain, largely innervating regions involved in generation/ modulation 
of hippocampal theta rhythm; and we demonstrated that these neurons display phase-locked firing with theta oscillations. Thus, we propose that the NI is a key site of CRF action, with significant capacity to modulate cognitive processes in response to neurogenic stressors. 


\section{References}

Aguilera G \& Liu Y (2012). The molecular physiology of CRH neurons. Front Neuroendocrinol 33, 67-84.

Alon T, Zhou L, Perez CA, Garfield AS, Friedman JM \& Heisler LK (2009). Transgenic mice expressing green fluorescent protein under the control of the corticotropinreleasing hormone promoter. Endocrinology 150, 5626-5632.

Banerjee A, Shen PJ, Ma S, Bathgate RAD \& Gundlach AL (2010). Swim stress excitation of nucleus incertus and rapid induction of relaxin-3 expression via CRF1 activation. Neuropharmacology 58, 145-155.

Bathgate RAD, Samuel CS, Burazin TCD, Layfield S, Claasz AA, Reytomas IG, Dawson NF, Zhao C, Bond C, Summers RJ, Parry LJ, Wade JD \& Tregear GW (2002). Human relaxin gene $3(\mathrm{H} 3)$ and the equivalent mouse relaxin (M3) gene. Novel members of the relaxin peptide family. J Biol Chem 277, 1148-1157.

Bienvenu TC, Busti D, Magill PJ, Ferraguti F \& Capogna M (2012). Cell-type-specific recruitment of amygdala interneurons to hippocampal theta rhythm and noxious stimuli in vivo. Neuron 74, 1059-1074.

Bittencourt JC \& Sawchenko PE (2000). Do centrally administered neuropeptides access cognate receptors? An analysis in the central corticotropin-releasing factor system. $J$ Neurosci 20, 1142-1156.

Burazin TCD, Bathgate RAD, Macris M, Layfield S, Gundlach AL \& Tregear GW (2002). Restricted, but abundant, expression of the novel rat gene-3 (R3) relaxin in the dorsal tegmental region of brain. J Neurochem 82, 1553-1557.

Calaresu FR \& Mogenson GJ (1972). Cardiovascular responses to electrical stimulation of the septum in the rat. Am J Physiol 223, 777-782.

Callander GE, Ma S, Ganella DE, Wimmer VC, Gundlach AL, Thomas WG \& Bathgate RA (2012). Silencing relaxin-3 in nucleus incertus of adult rodents: a viral vector-based approach to investigate neuropeptide function. PLoS One 7, e42300.

Cervera-Ferri A, Guerrero-Martinez J, Bataller-Mompean M, Taberner-Cortes A, MartinezRicos J, Ruiz-Torner A \& Teruel-Marti V (2011). Theta synchronization between the hippocampus and the nucleus incertus in urethane-anesthetized rats. Exp Brain Res 211, 177-192.

Cervera-Ferri A, Rahmani Y, Martinez-Bellver S, Teruel-Marti V \& Martinez-Ricos J (2012). Glutamatergic projection from the nucleus incertus to the septohippocampal system. Neurosci Lett 517, 71-76. 
Chen Y, Brunson KL, Muller MB, Cariaga W \& Baram TZ (2000). Immunocytochemical distribution of corticotropin-releasing hormone receptor type-1 (CRF(1))-like immunoreactivity in the mouse brain: light microscopy analysis using an antibody directed against the C-terminus. J Comp Neurol 420, 305-323.

Cottone P, Sabino V, Roberto M, Bajo M, Pockros L, Frihauf JB, Fekete EM, Steardo L, Rice KC, Grigoriadis DE, Conti B, Koob GF \& Zorrilla EP (2009). CRF system recruitment mediates dark side of compulsive eating. Proc Natl Acad Sci USA 106, 20016-20020.

Davson H (1969) The Cerebrospinal Fluid. In: Handbook of Neurochemistry, vol. 2 (Lajtha, A., ed), pp 23-48 New York: Plenum Press.

Drummond GB (2009). Reporting ethical matters in the Journal of Physiology: standards and advice. J Physiol 587, 713-719.

Elam M, Svensson TH \& Thoren P (1986). Locus coeruleus neurons and sympathetic nerves: activation by cutaneous sensory afferents. Brain Res 366, 254-261.

Espana RA \& Scammell TE (2011). Sleep neurobiology from a clinical perspective. Sleep 34, 845-858.

Fuentealba P, Begum R, Capogna M, Jinno S, Marton LF, Csicsvari J, Thomson A, Somogyi P \& Klausberger T (2008). Ivy cells: a population of nitric-oxide-producing, slowspiking GABAergic neurons and their involvement in hippocampal network activity. Neuron 57, 917-929.

Fuller PM, Sherman D, Pedersen NP, Saper CB \& Lu J (2011). Reassessment of the structural basis of the ascending arousal system. J Comp Neurol 519, 933-956.

Goto M, Swanson LW \& Canteras NS (2001). Connections of the nucleus incertus. J Comp Neurol 438, 86-122.

Huang Q, Timofeeva E \& Richard D (2006). Regulation of corticotropin-releasing factor and its types 1 and 2 receptors by leptin in rats subjected to treadmill running-induced stress. J Endocrinol 191, 179-188.

Justice NJ, Yuan ZF, Sawchenko PE \& Vale W (2008). Type 1 corticotropin-releasing factor receptor expression reported in $\mathrm{BAC}$ transgenic mice: implications for reconciling ligand-receptor mismatch in the central corticotropin-releasing factor system. J Comp Neurol 511, 479-496.

Kalin NH, Takahashi LK \& Chen FL (1994). Restraint stress increases corticotropinreleasing hormone mRNA content in the amygdala and paraventricular nucleus. Brain $\operatorname{Res}$ 656, 182-186. 
Kirby LG, Freeman-Daniels E, Lemos JC, Nunan JD, Lamy C, Akanwa A \& Beck SG (2008). Corticotropin-releasing factor increases GABA synaptic activity and induces inward current in 5-hydroxytryptamine dorsal raphe neurons. J Neurosci $\mathbf{2 8}$, 1292712937.

Kizawa H, Nishi K, Ishibashi Y, Harada M, Asano T, Ito Y, Suzuki N, Hinuma S, Fujisawa Y, Onda H, Nishimura O \& Fujino M (2003). Production of recombinant human relaxin 3 in AtT20 cells. Regul Pept 113, 79-84.

Koob GF \& Bloom FE (1985). Corticotropin-releasing factor and behavior. Fed Proc 44, 259-263.

Kubota Y, Inagaki S, Shiosaka S, Cho HJ, Tateishi K, Hashimura E, Hamaoka T \& Tohyama M (1983). The distribution of cholecystokinin octapeptide-like structures in the lower brain stem of the rat: an immunohistochemical analysis. Neuroscience 9, 587-604.

Lipski J (1981). Antidromic activation of neurones as an analytic tool in the study of the central nervous system. J Neurosci Meth 4, 1-32.

Lu J, Sherman D, Devor M \& Saper CB (2006). A putative flip-flop switch for control of REM sleep. Nature 441, 589-594.

Ma S, Bonaventure P, Ferraro T, Shen P-J, Burazin TCD, Bathgate RAD, Liu C, Tregear GW, Sutton SW \& Gundlach AL (2007). Relaxin-3 in GABA projection neurons of nucleus incertus suggests widespread influence on forebrain circuits via G-proteincoupled receptor-135 in the rat. Neuroscience 144, 165-190.

Ma S, Olucha-Bordonau FE, Hossain MA, Lin F, Kuei C, Liu C, Wade JD, Sutton SW, Nunez A \& Gundlach AL (2009a). Modulation of hippocampal theta oscillations and spatial memory by relaxin-3 neurons of the nucleus incertus. Learn Mem 16, 730-742.

Ma S, Sang Q, Lanciego JL \& Gundlach AL (2009b). Localization of relaxin-3 in brain of Macaca fascicularis: Identification of a nucleus incertus in primate. J Comp Neurol 517, 856-872.

Mallet N, Micklem BR, Henny P, Brown MT, Williams C, Bolam JP, Nakamura KC \& Magill PJ (2012). Dichotomous organization of the external globus pallidus. Neuron 74, 1075-1086.

Miklos IH \& Kovacs KJ (2002). GABAergic innervation of corticotropin-releasing hormone $(\mathrm{CRH})$-secreting parvocellular neurons and its plasticity as demonstrated by quantitative immunoelectron microscopy. Neuroscience 113, 581-592. 
Minkels RF, Juch PJ, Ter Horst GJ \& Van Willigen JD (1991). Projections of the parvocellular reticular formation to the contralateral mesencephalic trigeminal nucleus in the rat. Brain Res 547, 13-21.

Miyamoto Y, Watanabe Y \& Tanaka M (2008). Developmental expression and serotonergic regulation of relaxin 3/INSL7 in the nucleus incertus of rat brain. Regul Pept 145, 5459.

Moruzzi G \& Magoun HW (1949). Brain stem reticular formation and activation of the EEG. Electroencephalogr Clin Neurophysiol 1, 455-473.

Nishiyama M, Makino S, Iwasaki Y, Tanaka Y, Nazarloo HP, Kaneda T, Asaba K \& Hashimoto K (2008). CRH mRNA expression in the hypothalamic paraventricular nucleus is inhibited despite the activation of the hypothalamo-pituitary-adrenal axis during starvation. Brain Res 1228, 107-112.

Nunez A, Cervera-Ferri A, Olucha-Bordonau F, Ruiz-Torner A \& Teruel V (2006). Nucleus incertus contribution to hippocampal theta rhythm generation. Eur J Neurosci 23, 2731-2738.

Olucha-Bordonau FE, Otero-Garcia M, Sanchez-Perez AM, Nunez A, Ma S \& Gundlach AL (2012). Distribution and targets of the relaxin-3 innervation of the septal area in the rat. J Comp Neurol 520, 1903-1939.

Olucha-Bordonau FE, Teruel V, Barcia-Gonzalez J, Ruiz-Torner A, Valverde-Navarro AA \& Martinez-Soriano F (2003). Cytoarchitecture and efferent projections of the nucleus incertus of the rat. J Comp Neurol 464, 62-97.

Olucha F, Martinez-Garcia F \& Lopez-Garcia C (1985). A new stabilizing agent for the tetramethyl benzidine (TMB) reaction product in the histochemical detection of horseradish peroxidase (HRP). J Neurosci Meth 13, 131-138.

Paxinos G \& Watson C (1998) The Rat Brain in Stereotaxic Coordinates. San Diego: Academic Press.

Pinault D (1996). A novel single-cell staining procedure performed in vivo under electrophysiological control: morpho-functional features of juxtacellularly labeled thalamic cells and other central neurons with biocytin or Neurobiotin. $J$ Neurosci Meth 65, 113-136.

Potter E, Sutton S, Donaldson C, Chen R, Perrin M, Lewis K, Sawchenko PE \& Vale W (1994). Distribution of corticotropin-releasing factor receptor mRNA expression in the rat brain and pituitary. Proc Natl Acad Sci USA 91, 8777-8781. 
Punn A, Levine MA \& Grammatopoulos DK (2006). Identification of signaling molecules mediating corticotropin-releasing hormone-R1alpha-mitogen-activated protein kinase (MAPK) interactions: the critical role of phosphatidylinositol 3-kinase in regulating ERK1/2 but not p38 MAPK activation. Mol Endocrinol 20, 3179-3195.

Refojo D, Schweizer M, Kuehne C, Ehrenberg S, Thoeringer C, Vogl AM, Dedic N, Schumacher M, von Wolff G, Avrabos C, Touma C, Engblom D, Schutz G, Nave KA, Eder M, Wotjak CT, Sillaber I, Holsboer F, Wurst W \& Deussing JM (2011). Glutamatergic and dopaminergic neurons mediate anxiogenic and anxiolytic effects of CRHR1. Science 333, 1903-1907.

Ryan PJ, Ma S, Olucha-Bordonau FE \& Gundlach AL (2011). Nucleus incertus - an emerging modulatory role in arousal, stress and memory. Neurosci Biobehav Rev 35, 1326-1341.

Sawchenko PE \& Swanson LW (1985). Localization, colocalization, and plasticity of corticotropin-releasing factor immunoreactivity in rat brain. Fed Proc 44, 221-227.

Smith CM, Hosken IT, Sutton SW, Lawrence AJ \& Gundlach AL (2012). Relaxin-3 null mutation mice display a circadian hypoactivity phenotype. Genes Brain Behav 11, 94104.

Smith CM, Ryan PJ, Hosken IT, Ma S \& Gundlach AL (2011). Relaxin-3 systems in the brain - the first 10 years. J Chem Neuroanat 42, 262-275.

Smith CM, Shen P-J, Banerjee A, Bonaventure P, Ma S, Bathgate RAD, Sutton SW \& Gundlach AL (2010). Distribution of relaxin-3 and RXFP3 within arousal, stress, affective, and cognitive circuits of mouse brain. J Comp Neurol 518, 4016-4045.

Stamp J \& Herbert J (2001). Corticosterone modulates autonomic responses and adaptation of central immediate-early gene expression to repeated restraint stress. Neuroscience 107, 465-479.

Streeter GL (1903). Anatomy of the floor of the fourth ventricle. Am J Anat 2, 299-313.

Sutin EL \& Jacobowitz DM (1988). Immunocytochemical localization of peptides and other neurochemicals in the rat laterodorsal tegmental nucleus and adjacent area. J Comp Neurol 270, 243-270.

Tanaka M, Iijima N, Miyamoto Y, Fukusumi S, Itoh Y, Ozawa H \& Ibata Y (2005). Neurons expressing relaxin 3/INSL 7 in the nucleus incertus respond to stress. Eur J Neurosci 21, 1659-1670. 
Teruel-Marti V, Cervera-Ferri A, Nunez A, Valverde-Navarro AA, Olucha-Bordonau FE \& Ruiz-Torner A (2008). Anatomical evidence for a ponto-septal pathway via the nucleus incertus in the rat. Brain Res 1218, 87-96.

Van Pett K, Viau V, Bittencourt JC, Chan RK, Li HY, Arias C, Prins GS, Perrin M, Vale W \& Sawchenko PE (2000). Distribution of mRNAs encoding CRF receptors in brain and pituitary of rat and mouse. J Comp Neurol 428, 191-212.

Watanabe Y, Tsujimura A, Takao K, Nishi K, Ito Y, Yasuhara Y, Nakatomi Y, Yokoyama C, Fukui K, Miyakawa T \& Tanaka M (2011). Relaxin-3-deficient mice showed slight alteration in anxiety-related behavior. Front Behav Neurosci 5, 50.

Young C, Huang Y, Lin C, Shen Y \& Gean P (2001). Selective enhancement of 1-type calcium currents by corticotropin in acutely isolated rat amygdala neurons. $\mathrm{Mol}$ Pharmacol 59, 604-611.

Yu B \& Shinnick-Gallagher P (1998). Corticotropin-releasing factor increases dihydropyridine- and neurotoxin-resistant calcium currents in neurons of the central amygdala. J Pharmacol Exp Ther 284, 170-179.

Zar JH (2010) Biostatistical Analysis ( $5^{\text {th }}$ Edition). Upper Saddle River, New Jersey: Prentice Hall. 


\section{Author Contributions}

S.M., A.J.M.V. and A.L.G. conceived the project and all authors assisted in the design of the experiments - specifically, S.M. and A.J.M.V. designed, performed and analysed the in vivo electrophysiology and immunohistochemistry experiments; A.B. designed, performed and analysed the in vitro electrophysiology experiments; F.E.O. designed, performed and analysed the neural tract-tracing experiments. S.M. and A.L.G. collated results and wrote the article, and all authors revised the article critically and approved the final version.

\section{Acknowledgements}

This work was supported by research grants from the National Health and Medical Research Council (NHMRC) of Australia (520299 to S.M.; 277609, 509246 and 1005988 to A.L.G.; 350207 to A.J.M.V.; a research grant from the Polish Ministry of Science and Higher Education N N303 569939 (to A.B. and A.L.G.); a Commonwealth of Australia Endeavour Research Fellowship (to A.B.); a grant from the Florey Neuroscience Institutes Foundation (to S.M. and A.L.G.); research grants from the National Heart Foundation of Australia and Austin Hospital Foundation (to A.J.M.V.); a research grant from The Ministry of Health, Spain (ISCIII-FIS PI061816 to F.E.O.); a collaborative research grant from Johnson \& Johnson Pharmaceutical Research \& Development, LLC, San Diego, USA (to A.L.G.); a grant from the Besen Family Foundation and the Pratt Foundation (to A.L.G.); and by the Victorian Government Operational Infrastructure Support Program. S.M. was the recipient of an NHMRC (Australia) Biomedical Postdoctoral Fellowship. A.L.G. is an NHMRC (Australia) Senior Research Fellow. We thank Dr. Thomas Bienvenu for assistance with circular statistical analyses and Celia Pereira and Fabio Santos for technical assistance with the tract-tracing studies. 
Tables

Table 1. Effect of CRF on the firing of NI neurons and their RLN3 phenotype

\begin{tabular}{lccccccccccc}
\hline Response to CRF & \multicolumn{3}{c}{ Activated } & \multicolumn{3}{c}{ Inhibited } & \multicolumn{3}{c}{ Unaffected } \\
Response to MS stim & AD & OD & Sp & AD & OD & Sp & AD & OD & Sp \\
RLN3+ve $(\mathrm{n}=9)$ & 5 & 3 & 1 & 0 & 0 & 0 & 0 & 0 & 0 \\
RLN3-ve $(\mathrm{n}=13)$ & 0 & 0 & 1 & 1 & 5 & 2 & 0 & 0 & 4 \\
\hline
\end{tabular}

Values are the number of recorded NI neurons verified for the presence or absence of RLN3immunoreactivity following juxtacellular labelling. AD, antidromic; OD, orthodromic; $\mathrm{Sp}$, neither AD nor OD, but spontaneously firing. 
Figure 1. Locations of recorded and juxtacellularly-labelled neurons in the nucleus incertus that responded to $\mathbf{C R F}$. Anatomical structures labelled according to the classification of Paxinos \& Watson (1998). 4V, fourth ventricle; LC, locus coeruleus; Me5, mesencephalic nucleus of the trigeminal nerve; NIc, NI pars compacta; NId, NI pars dissipata; PDTg, posterodorsal tegmental nucleus. Scale bar $=0.5 \mathrm{~cm}$.

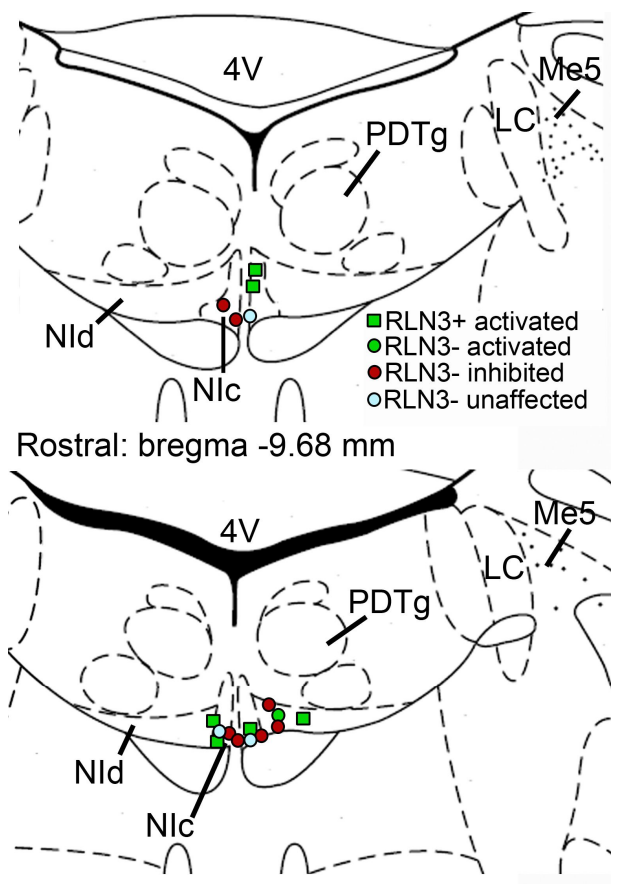

Mid: bregma $-9.80 \mathrm{~mm}$

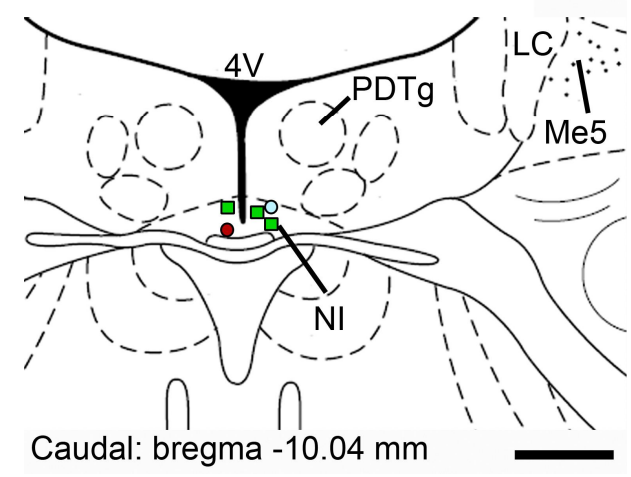


Figure 2. Response of a representative NI neuron to icv CRF in vivo that was RLN3immunopositive. $A$, application of $3 \mu \mathrm{g}$ CRF icv (grey shaded column) significantly increased the discharge rate of the neuron by $\sim 350 \%$, with onset of effect at 130 s postinfusion. There was little change in arterial blood pressure following icv CRF infusion. $B$, immunofluorescent visualisation of the recorded and juxtacellularly-labelled neuron revealed it was RLN3-positive. $C$, four superimposed traces in which a spontaneous spike (AP) triggered an electrical stimulus in the medial septum (Stim; mean $10.91 \pm 0.06 \mathrm{~ms}$ ) that elicited a constant latency antidromic spike (AD; upper trace; mean $9.30 \pm 0.11 \mathrm{~ms}$ ). Spike collision (lower trace) when the stimulation was applied within the critical interval (mean $9.02 \pm 0.04 \mathrm{~ms}$ ), indicates that this neuron projects to the medial septum. $D$, the recorded neuron had a short spike duration of $0.63 \mathrm{~ms}$. E, light microscopic reconstruction (63× magnification) of a biotinamide-labelled RLN3 neuron that was activated by CRF. The location of the neuron is within the boundaries of the NI. Soma (black), dendrites (blue) and an axon (red) are drawn from 5 sections of $50 \mu \mathrm{m}$ thickness. Juxtacellular-labelling revealed the neuron expressed RLN3 (inset). Scale bar in photomicrographs of $B=20 \mu \mathrm{m}$ and $E=15$ $\mu \mathrm{m} .4 \mathrm{~V}$, fourth ventricle; mlf, medial longitudinal fasciculus; NIc, NI pars compacta; NId, NI pars dissipata; PDTg, posterodorsal tegmental nucleus.
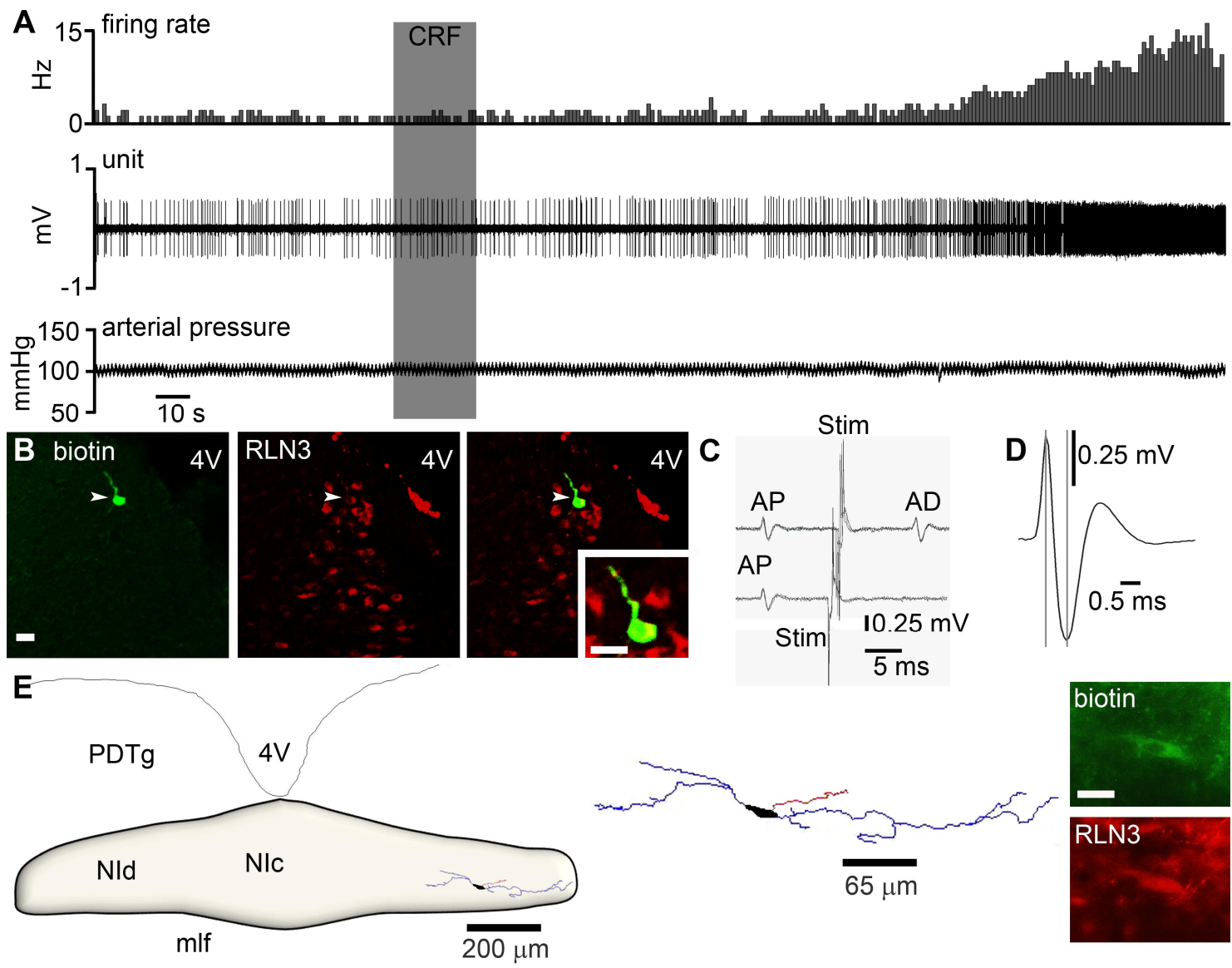
Figure 3. Response of a representative NI neuron to icv CRF in vivo that was RLN3immunonegative. $A$, application of $3 \mu \mathrm{g} C R F$ icv (grey shaded column) significantly decreased the discharge rate of the neuron by $\sim 50 \%$, with onset of effect at 140 s postinfusion. Arterial blood pressure increased by $\sim 15 \mathrm{~mm} \mathrm{Hg}$ following icv CRF infusion. $B$, immunofluorescent visualisation of the recorded and juxtacellularly-labelled neuron revealed it was RLN3-negative. $C$, peri-stimulus time histogram (100 sweeps) of orthodromic stimulation of firing elicited by medial septum stimulation $(*)$, indicates that the recorded neuron receives excitatory projections from the medial septum, with a delay of $\sim 17 \mathrm{~ms}$. $D$, the recorded neuron had a short spike duration of $0.60 \mathrm{~ms}$. $E$, light microscopic reconstruction ( $63 \times$ magnification) of a biotinamide-labelled non-RLN3 neuron that was inhibited by CRF. The location of the neuron is within the boundaries of the NI. Soma (black), dendrites (blue) and an axon (red) are drawn from 6 sections of $50 \mu \mathrm{m}$ thickness. Juxtacellular-labelling revealed the neuron lacked RLN3 (inset). Scale bar in photomicrographs of $B=20 \mu \mathrm{m}$ and $E=15 \mu \mathrm{m} .4 \mathrm{~V}$, fourth ventricle. $4 \mathrm{~V}$, fourth ventricle; mlf, medial longitudinal fasciculus; NIc, NI pars compacta; NId, NI pars dissipata; PDTg, posterodorsal tegmental nucleus.

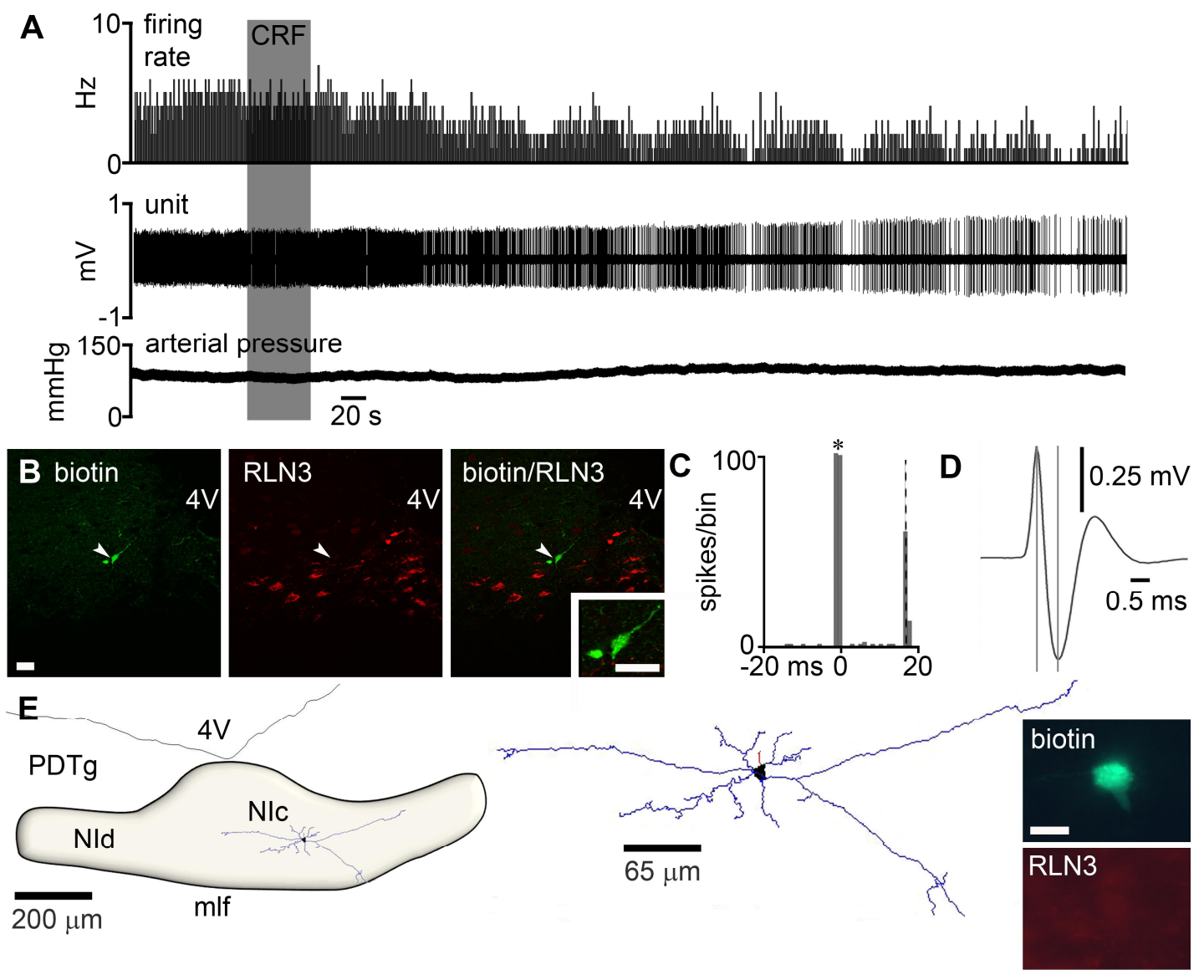


Figure 4. Firing rates of recorded NI neurons and their response to $\mathrm{CRF}$ in vivo $(\mathrm{n}=38$ rats, $\mathbf{n = 4 3}$ neurons). Neurons confirmed by juxtacellular-labelling as RLN3-positive are indicated by coloured squares, RLN3-negative by coloured circles and unlabelled neurons by grey circles. Because there were no significant differences between labelled and unlabelled neurons in all parameters measured, data was pooled for each group $(P=0.9$, one way ANOVA). $A$, the firing rate of 18 NI neurons was significantly increased by $\sim 250 \%$ following icv CRF infusion ( $P<0.001$, paired $t$-test, $\mathrm{n}=18$ neurons). One RLN3 neuron with firing rate $>30 \mathrm{~Hz}$ was omitted for illustrative clarity. The firing rate of $19 \mathrm{NI}$ neurons was significantly decreased by $\sim 50 \%$ following icv CRF infusion $(P<0.001$, paired $t$-test, $\mathrm{n}=19$ neurons). One non-RLN3 neuron with firing rate $>20 \mathrm{~Hz}$ was omitted for illustrative clarity. $B$, no significant difference was observed in the basal firing rates of CRF activated, inhibited, or unaffected $(\mathrm{n}=6)$ NI neurons prior to CRF infusion $(P=0.8$, one way ANOVA) or in $(C)$ the time to onset of CRF effects between activated and inhibited neurons $(P=0.2$, unpaired $t$ test).
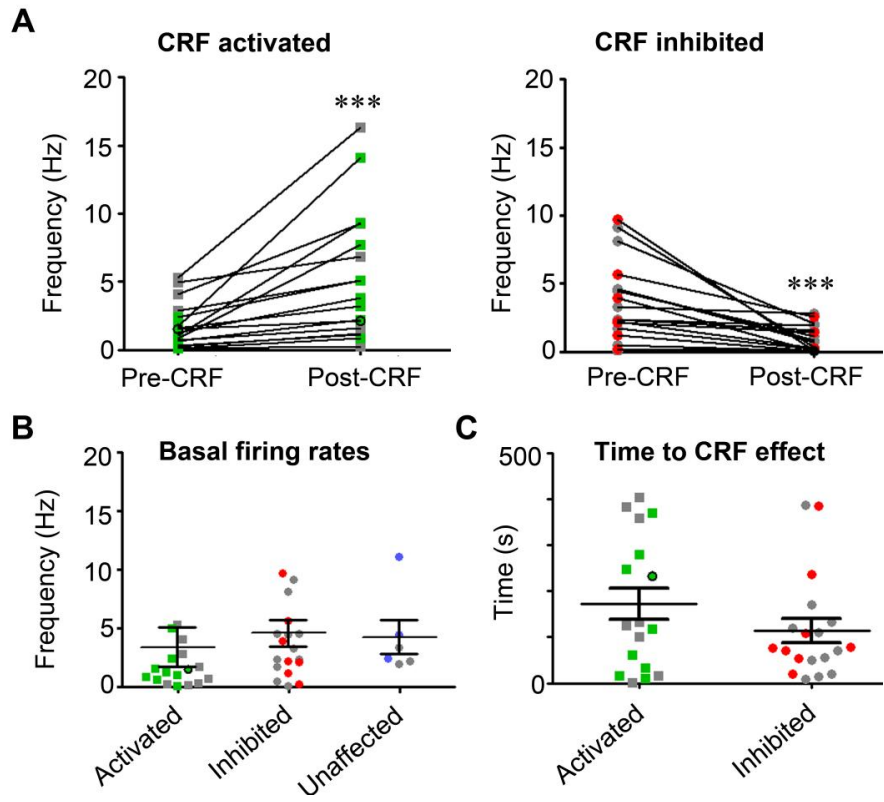
Figure 5. CRF excitation of NI neurons in vitro. $A$, a representative trace of current-clamp recording demonstrating the effects of bath applied CRF (100 nM, horizontal bar) on activity of an NI neuron, shown as a change in firing frequency ( $1 \mathrm{~s}$ bins, upper trace) and corresponding raw signal (lower trace) with amplified segments below. $B$, overlay of the raw signal recorded from the CRF activated neuron before (black) and after (red) CRF treatment, revealing an increase in action potential firing frequency and decrease in AHP peak and duration after $\mathrm{CRF}$ treatment. $C$, dose-dependent change in membrane potential following CRF-induced depolarisation. Values are presented as mean $\pm \mathrm{SEM}$ and the number of neurons tested for each concentration is indicated on each bar. $D$, current-clamp recording demonstrating the effect of three consecutive CRF applications (100 nM, horizontal bars) illustrating the sustained sensitivity of NI neurons to multiple CRF stimulations. $E$, membrane potential depolarisation of an NI neuron following $100 \mathrm{nM}$ CRF application in the presence of TTX $(1 \mu \mathrm{M})$, and a failure to depolarise to a second application of CRF applied in the presence of the CRF-R1 antagonist NBI 35965 (1 $\mu \mathrm{M}$, lower horizontal bar). $F$, confocal projection image ( $1 \mu \mathrm{m}$ optical slice) of a recorded, CRF-activated neuron confirmed that it was RLN3-positive. G, photomicrograph of a biocytin-filled RLN3 neuron in the NI pars compacta of a $250 \mu \mathrm{m}$ brain slice. 


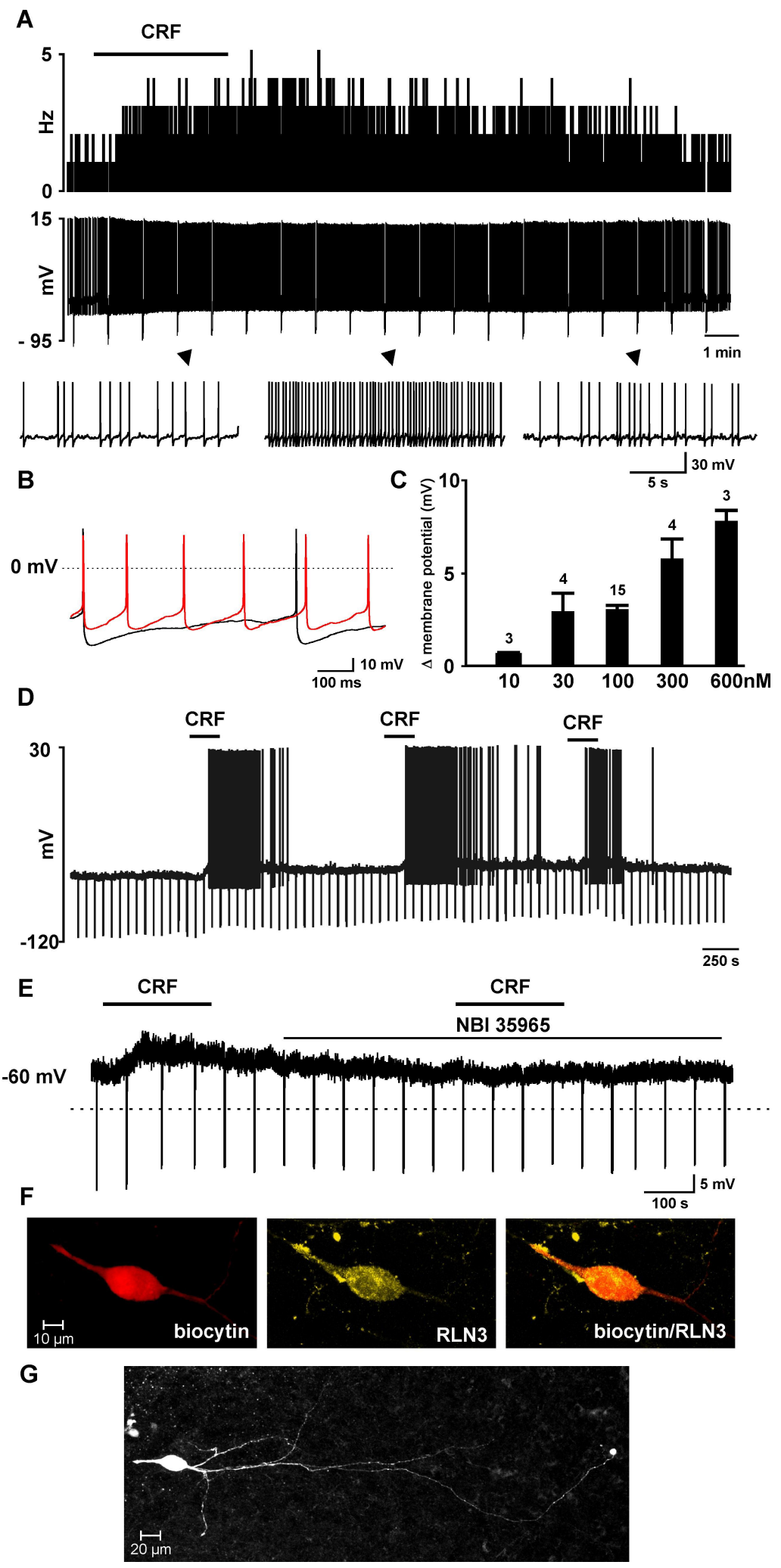


Figure 6. RLN3 NI neurons display coherent firing phase-locked to theta oscillations. $A$, Average phase histograms of RLN3 and non-RLN3 neurons ( $n=6$ each; mean \pm SEM) illustrating the strong modulation of RLN3 neurons in phase with theta oscillations, preferentially at the initial ascending phase, in contrast to non-RLN3 neurons. Two theta cycles are represented, where $0^{\circ}, 360^{\circ}$ and $720^{\circ}$ indicate oscillation troughs. $B$, raw trace of single unit activity of a RLN3-positive neuron recorded during hippocampal theta activity of the local field potential (LFP) illustrates spiking occurred preferentially during the ascending phase of theta oscillations. $C$, raw trace of single unit activity of a RLN3 neuron shows burstlike firing during theta oscillations. $D$, Polar plot of individual neurons' preferred theta phases and modulation depths (RLN3 and non-RLN3 neurons indicated by green circles and red squares, respectively).
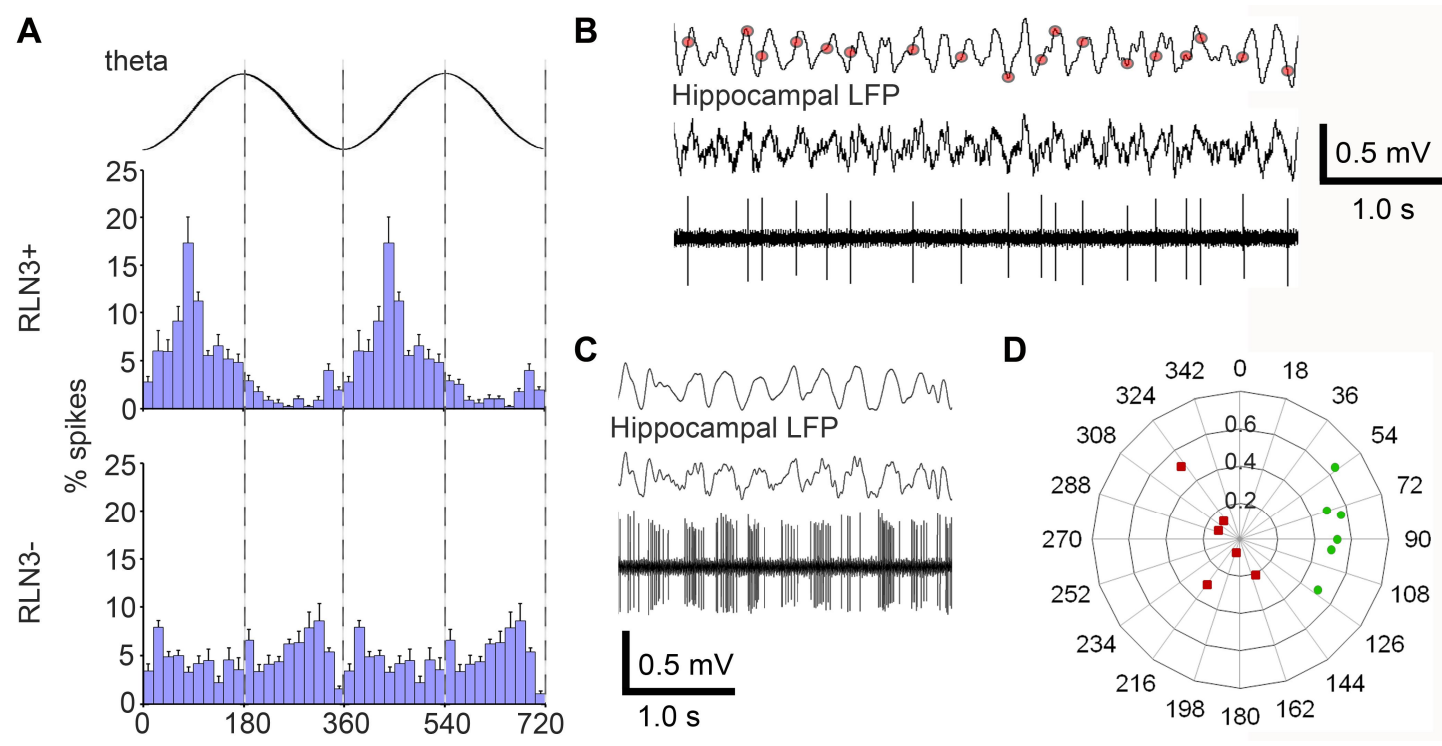
Figure 7. Localisation of CRF-R1 and RLN3 immunoreactivity (IR) in the rat nucleus incertus (NI). $A$, schematic depiction of the NI consisting of two midline columns of densely-packed, large neurons (NI pars compacta, NIc) and more loosely distributed neurons located laterally (NI pars dissipata, NId), which lie in the midline of the pontine central grey - ventral to the fourth ventricle and dorsal tegmental nucleus (DTg), and medial to locus coeruleus (LC) and mesencephalic nucleus of the trigeminal nerve (Me5) (Paxinos \& Watson 1998). $B$, cells of the NIc and NId are immunopositive for CRF-R1-IR (green) and RLN3-IR (red), though RLN3-IR is more prominently detected in NIc, whereas CRF-R1-IR is present in both NIc and NId $(n=5)$. All RLN3-immunoreactive cells contain CRF-R1-IR, but not all CRF-R1-immunoreactive cells contain RLN3-IR, particularly in the NId. $C$ - $E$, higher magnification images of a region between the NIc and NId outlined in B. $C$, CRF-R1-IR appears as punctate, ring-like staining, indicative of labelling of membrane-bound receptor. $D$, in contrast, RLN3-IR is concentrated within the cytoplasm, proximal process and fibers, reflecting the synthesis, storage and trafficking of the peptide. $E$, merge of images $B$ and $C$. Examples of CRF-R1-IR that did not co-localise with RLN3-IR are indicated by arrows in $C$ and $E$. F-H, localisation of CRF-R1 in NI neurons. $F$, strong NeuN-IR in NI neurons clearly delineates it from the DTg. $G$ and $H$, all CRF-R1-IR was associated with NeuN-IR and clear examples are indicated by arrows. Some NeuN-IR neurons that were CRF-R1-negative are indicated by arrowheads. Images are confocal microscope single optical sections. Scale bars: $A=1.2 \mathrm{~cm}$ and $B-H=20 \mu \mathrm{m} .4 \mathrm{~V}$, fourth ventricle; DTg, dorsal tegmental nucleus; LC, locus coeruleus; Me5, mesencephalic nucleus of the trigeminal nerve; PRNc, caudal pontine reticular nucleus. 

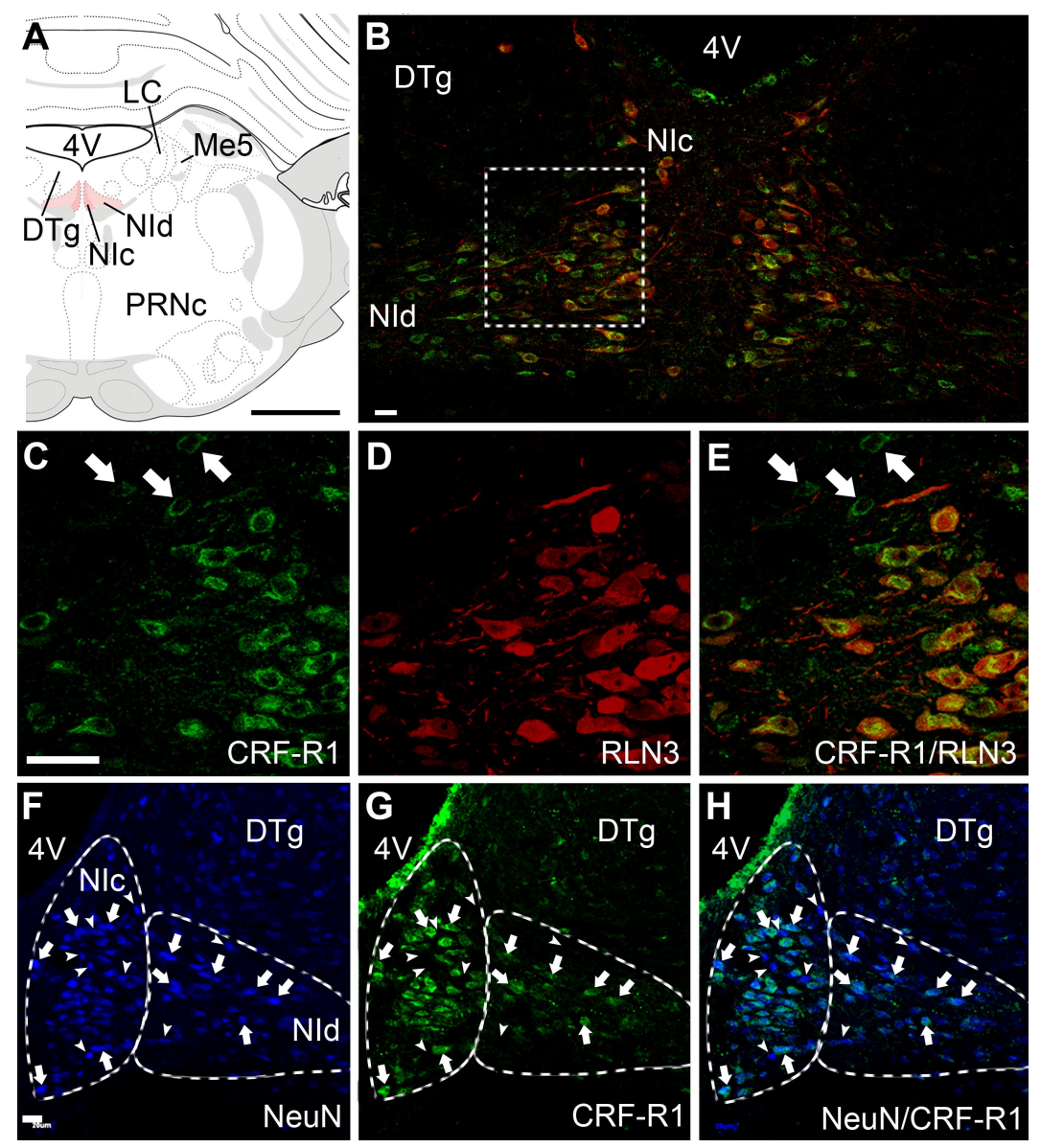
Figure 8. CRF neurons of the lateral preoptic area (LPO) project to the NI. $A$, distribution map of single- and double-labelling for FG and CRF in the preoptic hypothalamus $(B)$ following FG injection into the NI $(\mathrm{n}=5) . C$, photomicrograph of retrogradely-transported FG (stained dark blue with TMB-AHM, black arrowheads) colocalized in a subset of CRF neurons (stained brown with DAB, black arrows) in the lateral preoptic area. CRF neurons not retrogradely-labeled are indicated by white arrows. Scale bars: $A=0.5 \mathrm{~mm} ; B=200 \mu \mathrm{m} ; C=20 \mu \mathrm{m} .4 \mathrm{~V}$, fourth ventricle; f, fornix; LPO, lateral preoptic nucleus; LV, lateral ventricle; MPA, medial preoptic area; MPO, medial preoptic nucleus; NI, nucleus incertus; ox, optic chiasm; PDTg, posterior dorsal tegmental nucleus; Pe, periventricular hypothalamic nucleus; PVN, paraventricular hypothalamic nucleus; SCh, suprachiasmatic nucleus; SO, supraoptic nucleus.

A

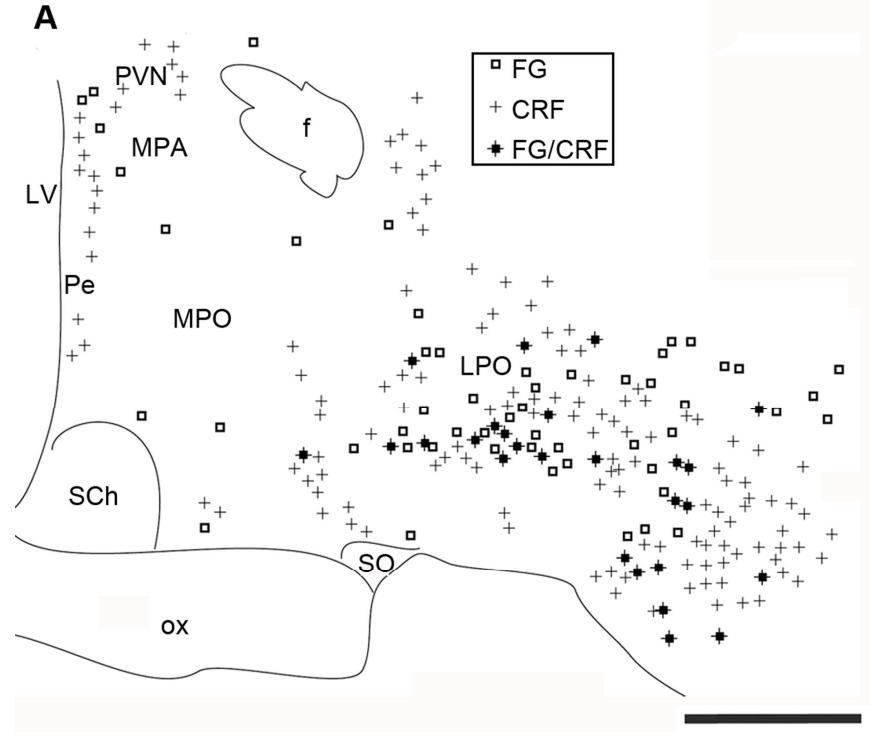

B

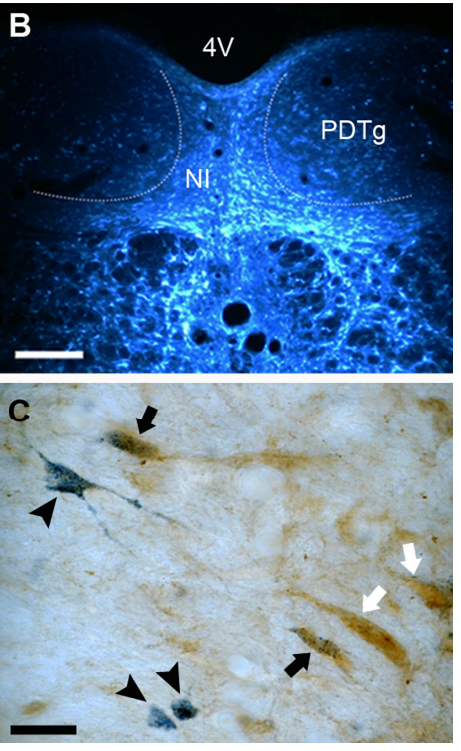


Figure 9. Model of CRF-R1 localisation and CRF actions in the NI. Schematic image of a CRF-R1 positive neuron and CRF-R1/RLN3 positive neuron (foreground), which together constitute approximately half of the total NI neuronal population. In vivo and in vitro electrophysiological recordings suggest that these CRF-R1 and CRF-R1/RLN3 neurons are directly activated by CRF via postsynaptic CRF-R1, resulting in increased firing. Furthermore, a population of RLN3-negative cells (background) are inhibited by CRF in vivo, an effect not observed in vitro, suggesting that these neurons may receive an inhibitory input from a distant source that is activated by CRF, which is disconnected in a brain slice preparation.

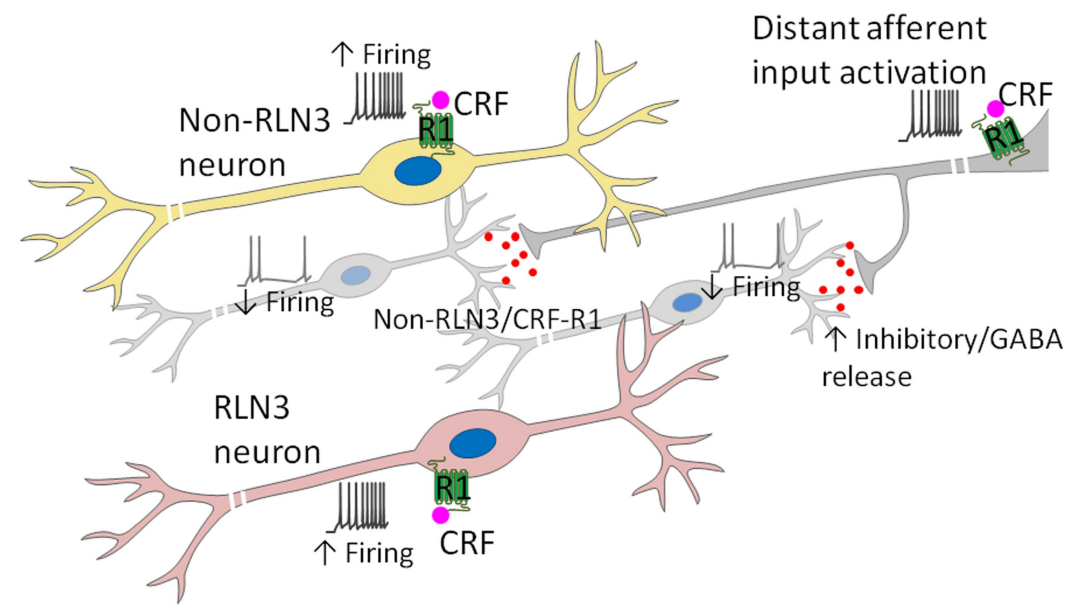




\section{University Library}

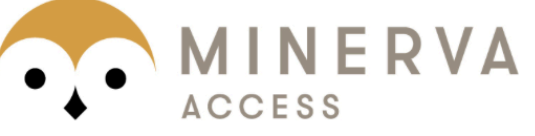

A gateway to Melbourne's research publications

Minerva Access is the Institutional Repository of The University of Melbourne

Author/s:

Ma, S;Blasiak, A;Olucha-Bordonau, FE;Verberne, AJM;Gundlach, AL

Title:

Heterogeneous responses of nucleus incertus neurons to corticotrophin-releasing factor and coherent activity with hippocampal theta rhythm in the rat

Date:

2013-08-15

Citation:

Ma, S., Blasiak, A., Olucha-Bordonau, F. E., Verberne, A. J. M. \& Gundlach, A. L. (2013). Heterogeneous responses of nucleus incertus neurons to corticotrophin-releasing factor and coherent activity with hippocampal theta rhythm in the rat. JOURNAL OF PHYSIOLOGYLONDON, 591 (16), pp.3981-4001. https://doi.org/10.1113/jphysiol.2013.254300.

Persistent Link:

http://hdl.handle.net/11343/44159 